\title{
AN A PRIORI ERROR ANALYSIS OF THE LOCAL DISCONTINUOUS GALERKIN METHOD FOR ELLIPTIC PROBLEMS
}

\author{
PAUL CASTILLO *, BERNARDO COCKBURN $\dagger$, ILARIA PERUGIA $\ddagger$, AND DOMINIK \\ SCHÖTZAU §
}

April 27, 2000

\begin{abstract}
In this paper, we present the first a priori error analysis for the Local Discontinuous Galerkin method for a model elliptic problem. For arbitrary meshes with hanging nodes and elements of various shapes, we show that, for stabilization parameters of order one, the $\mathrm{L}^{2}$-norm of the gradient and the $\mathrm{L}^{2}$-norm of the potential are of order $k$ and $k+1 / 2$, respectively, when polynomials of total degree at least $k$ are used; if stabilization parameters of order $h^{-1}$ are taken, the order of convergence of the potential increases to $k+1$. The optimality of these theoretical results are tested in a series of numerical experiments on two dimensional domains.
\end{abstract}

Key words. Finite elements, discontinuous Galerkin methods, elliptic problems

AMS subject classifications. $65 \mathrm{~N} 30$

1. Introduction. In this paper, we present the first a priori error analysis of the Local Discontinuous Galerkin (LDG) method for the following classical model elliptic problem:

$$
\begin{aligned}
-\Delta u & =f & & \text { in } \Omega, \\
u & =g_{\mathcal{D}} & & \text { on } \Gamma_{\mathcal{D}}, \\
\frac{\partial u}{\partial n} & =\boldsymbol{g}_{\mathcal{N}} \cdot \boldsymbol{n} & & \text { on } \Gamma_{\mathcal{N}},
\end{aligned}
$$

where $\Omega$ is a bounded domain of $\mathbb{R}^{d}$ and $\boldsymbol{n}$ is the outward unit normal to its boundary $\bar{\Gamma}_{\mathcal{D}} \cup \bar{\Gamma}_{\mathcal{N}}$; for the sake of simplicity, we assume that the $(d-1)$-dimensional measure of $\Gamma_{\mathcal{D}}$ is non-zero.

The LDG method was introduced by Cockburn and Shu in [25] as an extension to general convection-diffusion problems of the numerical scheme for the compressible Navier-Stokes equations proposed by Bassi and Rebay in [6]. This scheme was in turn an extension of the Runge-Kutta Discontinuous Galerkin (RKDG) method developed

${ }^{*}$ Scientific Computing Program, School of Mathematics, University of Minnesota, Vincent Hall, Minneapolis, MN 55455, email: castillo@math.umn.edu.

†School of Mathematics, University of Minnesota, Vincent Hall, Minneapolis, MN 55455, email: cockburn@math.umn.edu. Supported in part by the National Science Foundation (Grant DMS9806956) and by the University of Minnesota Supercomputing Institute.

†Dipartimento di Matematica, Università di Pavia, Via Ferrata 1, 27100 Pavia, Italy, email: perugia@dimat.unipv.it. Supported in part by the Consiglio Nazionale delle Ricerche. This work was carried out when the author was a Visiting Professor at the School of Mathematics, University of Minnesota.

$\S$ School of Mathematics, University of Minnesota, Vincent Hall, Minneapolis, MN 55455, email: schoetza@math. umn.edu. Supported by the Swiss National Science Foundation (Schweizerischer Nationalfonds). 
by Cockburn and Shu [19, 21, 23, 24, 26] for nonlinear hyperbolic systems. The LDG method is one of several discontinuous Galerkin methods which are being vigorously studied, especially as applied to convection-diffusion problems, because of their applicability to a wide range of problems, their properties of local conservativity and high degree of locality, and their flexibility in handling adaptive $h p$-refinement. The state of the art of the development of discontinuous Galerkin methods can be found in the volume [20] edited by Cockburn, Karniadakis and Shu.

Let us give the reader familiar with (classical and stabilized) mixed and mortar finite element methods for elliptic problems an idea of what kind of method is the LDG method.

- The LDG is obtained by using a space discretization that was originally applied to first-order hyperbolic systems. Hence, to define the method, we rewrite our elliptic model problem as a system of first-order equations and then we discretize it. Thus, we introduce the auxiliary variable $\boldsymbol{q}=\nabla u$ and obtain the equations

$$
\begin{aligned}
\boldsymbol{q} & =\nabla u & & \text { in } \Omega, \\
-\nabla \cdot \boldsymbol{q} & =f & & \text { in } \Omega, \\
u & =g_{\mathcal{D}} & & \text { on } \Gamma_{\mathcal{D}} \\
\boldsymbol{q} \cdot \boldsymbol{n} & =\boldsymbol{g}_{\mathcal{N}} \cdot \boldsymbol{n} & & \text { on } \Gamma_{\mathcal{N}} .
\end{aligned}
$$

Since these are nothing but the equations used to define classical mixed finite element methods, the LDG method can be considered as a mixed finite element method. However, the auxiliary variable $\boldsymbol{q}$ can be eliminated from the equations which is usually not the case for classical mixed methods.

- In the LDG method, local conservativity holds because the conservation laws (1.2) and (1.3) are weakly enforced element by element. In order to do that, suitable discrete approximations of the traces of the fluxes on the boundary of the elements are needed which are provided by the so-called numerical fluxes; these are widely used in the numerical approximation of non-linear hyperbolic conservation laws and are nothing but the so-called approximate Riemann solvers; see Cockburn [17]. As in the case of non-linear hyperbolic conservation laws, these numerical fluxes enhance the stability of the method and hence the quality of its approximation. This is why the LDG method is strongly related to stabilized mixed finite element methods; indeed, the stabilization is associated with the jumps of the approximate solution across the element boundaries.

- The LDG method allows general meshes with hanging nodes and elements of several shapes since no inter-element continuity is required. This is also a key property of the mortar finite element method. However, in the LDG method there are no Lagrange multipliers associated to the continuity constraint; instead, the Lagrange multiplier is replaced by fixed functions of the unknowns which are nothing but the above mentioned numerical fluxes.

- In the LDG method, on each element, both the approximation to $u$ as well as the approximations to each of the components of $\boldsymbol{q}$ belong to the same space, which is very convenient from an implementational point of view. Moreover, the lack of continuity constraints across element boundaries in the finite element spaces renders the coding of the $h p$-version of the LDG method much simpler than that of standard mixed methods.

Now, let us briefly describe the recent work on error analysis of DG methods in order to put our results into perspective. Analyses of the LDG method in the context of 
transient convection-diffusion problems have been carried out by Cockburn and Shu [25], by Cockburn and Dawson [18], by Castillo [14] and more recently by Castillo, Cockburn, Schötzau and Schwab [15].

The DG method of Baumann and Oden [7, 8, 9, 32] has also been analyzed by several authors. Oden, Babuška and Baumann [31] studied this method for one dimensional elliptic problems and later Wihler and Schwab [41] proved robust exponential rates of convergence of the Oden and Baumann DG method for stationary convectiondiffusion problems also in one space dimension. Rivière, Wheeler and Girault [35] and Rivière and Wheeler [34] analyzed several variations of the DG method of Baumann and Oden (involving interior penalty techniques) as applied to non-linear convectiondiffusion problems and, finally, Süli, Schwab and Houston [38] synthesized the selfadjoint elliptic, parabolic, and hyperbolic theory by extending the analysis of these DG methods to general second-order linear partial differential equations with nonnegative characteristic form.

As applied to purely elliptic problems, the LDG method and the method of Baumann and Oden are strongly related to the so-called interior interior penalty (IP) methods explored mainly by Babuška and Zlámal [3], Douglas and Dupont [27], Baker [4], Wheeler [39], Arnold [2] and later by Baker, Jureidini and Karakashian [5], by Rusten, Vassilevski, and Winther [36] and by Becker and Hansbo [10]. All of these DG methods for elliptic problems can be recast within a single framework as shown by Arnold, Brezzi, Cockburn and Marini [1]; this framework should provide a basis for a better understanding of the connections among them and lead to a unified error analysis of these methods. As a contribution to this effort, we present in this paper an a priori error analysis of the LDG method for purely elliptic problems.

We show that if polynomials of degree at least $k$ are used in all the elements, the rate of convergence of the LDG method in the $\mathrm{L}^{2}$-norm of $u$ and $\boldsymbol{q}$ are of order $k+1 / 2$ and $k$, respectively, when the stabilization or penalization parameter $C_{11}$ is taken to be of order one. When the stabilization parameter $C_{11}$ is taken to be of order $h^{-1}$, the order of convergence of $u$ is proven to be $k+1$, as expected. Indeed, this is what happens for the interior penalty methods and for the modifications of the method of Bassi and Rebay [6] studied by Brezzi, Manzini, Marini, Pietra and Russo [13]; the penalization parameters of these methods are also of order $h^{-1}$. These results are summarized in the Table 1.1.

TABLE 1.1

Orders of convergence for $k \geq 1$.

\begin{tabular}{cccc}
\hline method & penalization & $\left\|u-u_{N}\right\|_{0}$ & $\|\boldsymbol{q}-\boldsymbol{q}\|_{0}$ \\
\hline LDG & & & \\
LDG & $\mathcal{O}(1)$ & $k+1 / 2$ & $k$ \\
interior penalty & $\mathcal{O}\left(h^{-1}\right)$ & $k+1$ & $k$ \\
Brezzi et al. & $\mathcal{O}\left(h^{-1}\right)$ & $k+1$ & $k$ \\
& & & $k$ \\
\hline
\end{tabular}

Finally, let us point out that the order of convergence of $u$ for the DG method for purely convective problems is $k+1 / 2$. This order of convergence was proven by Johnson and Pitkäranta [30] and later confirmed by Peterson [33] to be sharp. Whether 
or not a similar phenomenon is actually taking place for the LDG method, with the stabilization parameter $C_{11}$ of order one, as applied to elliptic problems remains to be investigated. Our numerical experiments for the LDG method have all been performed on structured and unstructured triangulations without hanging nodes and give the optimal orders of convergence of $k+1$ and $k$ for $u$ and $\boldsymbol{q}$, respectively, with $C_{11}$ of order $h^{-1}$ and, remarkably, with $C_{11}$ of order one.

The organization of the paper is as follows. In section 2, we present the LDG methods and state and discuss our main a priori error estimates. We also give a brief sketch of the proofs in order to display the ideas of our analysis. The analysis is carried out in full detail in section 3 and several possible extensions are indicated in section 4 . In section 5, we present several numerical experiments testing the sharpness of our theoretical results. We end in section 6 with some concluding remarks.

2. The main results. In this section, we formulate the LDG method and show that it possesses a well-defined solution. We then state and discuss our main result and, finally, we display the main ideas of our error analysis.

We assume, to avoid unnecessary technicalities, that the exact solution $u$ of our model problem (1.1) belongs to $H^{2}(\Omega)$ and that the solution of the so-called adjoint problem satisfies the standard ellipticity regularity property. Extensions to more general situations are discussed in section 4 .

2.1. The LDG method. To introduce our LDG method, we consider a general discontinuous Galerkin (DG) method of which the LDG method is a particular but important case. We consider a general triangulation $\mathcal{T}$ with hanging nodes whose elements $K$ are of various shapes.

To obtain the weak formulation with which our DG method is defined, we multiply equations (1.2) and (1.3) by arbitrary, smooth test functions $r$ and $v$, respectively, and integrate by parts over the element $K \in \mathcal{T}$ to obtain

$$
\begin{aligned}
& \int_{K} \boldsymbol{q} \cdot \boldsymbol{r} d \boldsymbol{x}=-\int_{K} u \nabla \cdot \boldsymbol{r} d \boldsymbol{x}+\int_{\partial K} u \boldsymbol{r} \cdot \boldsymbol{n} d s, \\
& \int_{K} \boldsymbol{q} \cdot \nabla v d \boldsymbol{x}=\int_{K} f v d \boldsymbol{x}+\int_{\partial K} v \boldsymbol{q} \cdot \boldsymbol{n} d s .
\end{aligned}
$$

Note that the above equations are well defined for any functions $(\boldsymbol{q}, u)$ and $(\boldsymbol{r}, v)$ in $\boldsymbol{M} \times V$ where

$$
\begin{aligned}
& \boldsymbol{M}:=\left\{\boldsymbol{q} \in\left(L^{2}(\Omega)\right)^{d}:\left.\boldsymbol{q}\right|_{K} \in H^{1}(K)^{d}, \forall K \in \mathcal{T}\right\}, \\
& V:=\left\{u \in L^{2}(\Omega):\left.u\right|_{K} \in H^{1}(K), \forall K \in \mathcal{T}\right\} .
\end{aligned}
$$

Next, we seek to approximate the exact solution $(\boldsymbol{q}, u)$ with functions $\left(\boldsymbol{q}_{N}, u_{N}\right)$ in the finite element space $\boldsymbol{M}_{N} \times V_{N} \subset \boldsymbol{M} \times V$, where

$$
\begin{aligned}
& \boldsymbol{M}_{N}:=\left\{\boldsymbol{q} \in\left(L^{2}(\Omega)\right)^{d}:\left.\boldsymbol{q}\right|_{K} \in \mathcal{S}(K)^{d}, \forall K \in \mathcal{T}\right\}, \\
& V_{N}:=\left\{u \in L^{2}(\Omega):\left.u\right|_{K} \in \mathcal{S}(K), \forall K \in \mathcal{T}\right\},
\end{aligned}
$$

and the local finite element space $\mathcal{S}(K)$ typically consists of polynomials. Note that for a given element $K$, the restrictions to $K$ of $u_{N}$ and of each of the components of $\boldsymbol{q}_{N}$ belong to the same local space; this renders the coding of these methods considerably simpler than that of the standard mixed methods, especially for high-order polynomial 
local spaces. In order to ensure the existence of the approximate solution of the DG method, we require the following local and quite mild condition:

$$
v \in \mathcal{S}(K): \int_{K} \nabla v \cdot r d x=0 \quad \forall \boldsymbol{r} \in \mathcal{S}^{d}(K) \quad \text { implies } \quad \nabla v \equiv 0 \text { on } K .
$$

Other than these properties, there is complete freedom in the choice of the local spaces since no inter-element continuity is required at all.

The approximate solution $\left(\boldsymbol{q}_{N}, u_{N}\right)$ is then defined by using the above weak formulation, that is, by imposing that for all $K \in \mathcal{T}$, for all $(\boldsymbol{r}, v) \in \mathcal{S}(K)^{d} \times \mathcal{S}(K)$,

$$
\begin{aligned}
& \int_{K} \boldsymbol{q}_{N} \cdot \boldsymbol{r} d \boldsymbol{x}=-\int_{K} u_{N} \nabla \cdot \boldsymbol{r} d \boldsymbol{x}+\int_{\partial K} \widehat{u}_{N} \boldsymbol{r} \cdot \boldsymbol{n} d s, \\
& \int_{K} \boldsymbol{q}_{N} \cdot \nabla v d \boldsymbol{x}=\int_{K} f v d \boldsymbol{x}+\int_{\partial K} v \widehat{\boldsymbol{q}}_{N} \cdot \boldsymbol{n} d s,
\end{aligned}
$$

where the numerical fluxes $\widehat{u}_{N}$ and $\widehat{\boldsymbol{q}}_{N}$ have to be suitably defined in order to ensure the stability of the method and in order to enhance its accuracy.

As pointed out in the introduction, we can see that the numerical fluxes $\widehat{u}_{N}$ and $\widehat{\boldsymbol{q}}_{N}$ are nothing but discrete approximations to the traces of $u$ and $\boldsymbol{q}$ on the boundary of the elements. To define these numerical fluxes, let us first introduce some notation. Let $K^{+}$and $K^{-}$be two adjacent elements of $\mathcal{T}$; let $\boldsymbol{x}$ be an arbitrary point of the set $e=\partial K^{+} \cap \partial K^{-}$, which is assumed to have a non-zero $(d-1)$-dimensional measure, and let $\boldsymbol{n}^{+}$and $\boldsymbol{n}^{-}$be the corresponding outward unit normals at that point. Let $(\boldsymbol{q}, u)$ be a function smooth inside each element $K^{ \pm}$and let us denote by $\left(\boldsymbol{q}^{ \pm}, u^{ \pm}\right)$ the traces of $(\boldsymbol{q}, u)$ on $e$ from the interior of $K^{ \pm}$. Then, we define the mean values $\{[\cdot\}$ and jumps $\llbracket \cdot \rrbracket$ at $\boldsymbol{x} \in e$ as follows:

$$
\begin{array}{ll}
\{u\}:=\left(u^{+}+u^{-}\right) / 2, & \left\{\boldsymbol{q} \rrbracket:=\left(\boldsymbol{q}^{+}+\boldsymbol{q}^{-}\right) / 2,\right. \\
\llbracket u \rrbracket:=u^{+} \boldsymbol{n}^{+}+u^{-} \boldsymbol{n}^{-}, & \llbracket \boldsymbol{q} \rrbracket:=\boldsymbol{q}^{+} \cdot \boldsymbol{n}^{+}+\boldsymbol{q}^{-} \cdot \boldsymbol{n}^{-} .
\end{array}
$$

Note that the jump in $u$ is a vector and the jump in $\boldsymbol{q}$ is a scalar which only involves the normal component of $\boldsymbol{q}$.

We are now ready to introduce the expressions that define the numerical fluxes in (2.2) and (2.3). If the set $e$ is inside the domain $\Omega$, we take

$$
\left[\begin{array}{l}
\widehat{\boldsymbol{q}} \\
\widehat{u}
\end{array}\right]:=\left[\begin{array}{l}
\{\boldsymbol{q}\}\} \\
\{u u\}
\end{array}\right]-\left[\begin{array}{cc}
C_{11} & \boldsymbol{C}_{12} \\
-C_{12} & C_{22}
\end{array}\right]\left[\begin{array}{l}
\llbracket u \rrbracket \\
\llbracket \boldsymbol{q} \rrbracket
\end{array}\right],
$$

where the auxiliary parameters $C_{11}, C_{12}$ and $C_{22}$ depend on $\boldsymbol{x} \in e$ and are still at our disposal. The boundary conditions are imposed through a suitable definition of $(\widehat{\boldsymbol{q}}, \widehat{u})$, namely,

$$
\widehat{\boldsymbol{q}}:= \begin{cases}\boldsymbol{q}^{+}-C_{11}\left(u^{+} \boldsymbol{n}^{+}+g_{\mathcal{D}} \boldsymbol{n}^{-}\right) & \text {on } \Gamma_{\mathcal{D}} \\ \boldsymbol{g}_{\mathcal{N}} & \text { on } \Gamma_{\mathcal{N}}\end{cases}
$$

and

$$
\widehat{u}:= \begin{cases}g_{\mathcal{D}} & \text { on } \Gamma_{\mathcal{D}} \\ u^{+}-C_{22}\left(\boldsymbol{q}^{+} \cdot \boldsymbol{n}^{+}+\boldsymbol{q}_{\mathcal{N}} \cdot \boldsymbol{n}^{-}\right) & \text {on } \Gamma_{\mathcal{N}}\end{cases}
$$

where the superscript + denotes quantities related to the element the edge we are considering belongs to, and $\boldsymbol{n}^{-}=-\boldsymbol{n}^{+}$. We remark that the definition of $(\widehat{\boldsymbol{q}}, \widehat{u})$ on 
the boundary $\partial \Omega$ is still of the form (2.4) if the exterior traces $\left(\boldsymbol{q}^{-}, u^{-}\right)$are taken to be

$$
\left(\boldsymbol{q}^{-}, u^{-}\right)=\left(\boldsymbol{q}^{+}, g_{\mathcal{D}}\right) \text { on } \Gamma_{\mathcal{D}}, \quad\left(\boldsymbol{q}^{-}, u^{-}\right)=\left(\boldsymbol{g}_{\mathcal{N}}, u^{+}\right) \text {on } \Gamma_{\mathcal{N}},
$$

and $\boldsymbol{C}_{12}$ is chosen such that $\boldsymbol{C}_{12} \cdot \boldsymbol{n}^{-}=1 / 2$ on $\Gamma_{\mathcal{D}}$ and $\boldsymbol{C}_{12} \cdot \boldsymbol{n}^{+}=1 / 2$ on $\Gamma_{\mathcal{N}}$.

Let us stress once more that the numerical fluxes we just defined are nothing but a particular case of the so-called approximate Riemann solvers widely used in numerical schemes for non-linear hyperbolic conservation laws.

This completes the definition of our DG method. The LDG method is obtained when $C_{22} \equiv 0$; in this case, the function $\boldsymbol{q}_{N}$ can be locally solved in terms of $u_{N}$ and hence eliminated from the equations, as can be easily seen from (2.3). This local solvability gives its name to the LDG method.

That this DG method actually defines a unique approximate solution depends in a crucial way on the coefficients $C_{11}$ and $C_{22}$. Indeed, we have the following result.

Proposition 2.1 (Well posedness of the DG method). Consider the DG method defined by the weak formulation (2.2) and (2.3), and by the numerical fluxes in (2.4) and (2.5). If the coefficients $C_{11}$ are positive and the coefficients $C_{22}$ are non-negative, the DG method defines a unique approximate solution $\left(\boldsymbol{q}_{N}, u_{N}\right) \in \boldsymbol{M}_{N} \times V_{N}$.

Notice that the above result, which we prove in the next subsection, is independent of the auxiliary vector parameter $\boldsymbol{C}_{12}$. The choice $\boldsymbol{C}_{21}=-\boldsymbol{C}_{12}$ in (2.4) ensures symmetry and stability of the DG method. Finally, let us point out that the role of the auxiliary parameters $C_{11}$ and $C_{22}$ is to enhance the stability and hence the accuracy of the method.

2.2. The classical mixed setting. The study of our DG method is greatly facilitated if we recast its formulation in a classical mixed finite element setting. To do that, we need to introduce some notation. We denote by $\mathcal{E}_{i}$ the union of all interior faces of the triangulation $\mathcal{T}$, by $\mathcal{E}_{\mathcal{D}}$ the union of faces on $\Gamma_{\mathcal{D}}$, and by $\mathcal{E}_{\mathcal{N}}$ the union of faces on $\Gamma_{\mathcal{N}}$; we assume that $\bar{\Gamma}_{\mathcal{D}}=\cup_{e \in \mathcal{E}_{\mathcal{D}}} \bar{e}$ and $\bar{\Gamma}_{\mathcal{N}}=\cup_{e \in \mathcal{E}_{\mathcal{N}}} \bar{e}$.

Now, we sum equations (2.2) and (2.3) over all elements and obtain, after some simple manipulations, that the DG approximation $\left(\boldsymbol{q}_{N}, u_{N}\right)$ is the unique solution of the following variational problem: find $\left(\boldsymbol{q}_{N}, u_{N}\right) \in \boldsymbol{M}_{N} \times V_{N}$ such that

$$
\begin{aligned}
a\left(\boldsymbol{q}_{N}, \boldsymbol{r}\right)+b\left(u_{N}, \boldsymbol{r}\right) & =F(\boldsymbol{r}), \\
-b\left(v, \boldsymbol{q}_{N}\right)+c\left(u_{N}, v\right) & =G(v),
\end{aligned}
$$

for all $(\boldsymbol{r}, v) \in \boldsymbol{M}_{N} \times V_{N}$. Here, the bilinear forms $a, b$ and $c$ are given by

$$
\begin{aligned}
a(\boldsymbol{q}, \boldsymbol{r}) & :=\int_{\Omega} \boldsymbol{q} \cdot \boldsymbol{r} d \boldsymbol{x}+\int_{\mathcal{E}_{i}} C_{22} \llbracket \boldsymbol{q} \rrbracket \llbracket \boldsymbol{r} \rrbracket d s+\int_{\mathcal{E}_{\mathcal{N}}} C_{22}(\boldsymbol{q} \cdot \boldsymbol{n})(\boldsymbol{r} \cdot \boldsymbol{n}) d s, \\
b(u, \boldsymbol{r}) & :=\sum_{K \in \mathcal{T}} \int_{K} u \nabla \cdot \boldsymbol{r} d \boldsymbol{x}-\int_{\mathcal{E}_{i}}\left(\llbracket u \rrbracket+\boldsymbol{C}_{12} \cdot \llbracket u \rrbracket\right) \llbracket \boldsymbol{r} \rrbracket d s-\int_{\mathcal{E}_{\mathcal{N}}} u \boldsymbol{r} \cdot \boldsymbol{n} d s, \\
c(u, v) & :=\int_{\mathcal{E}_{i}} C_{11} \llbracket u \rrbracket \cdot \llbracket v \rrbracket d s+\int_{\mathcal{E}_{\mathcal{D}}} C_{11} u v d s .
\end{aligned}
$$

The linear forms $F, G$ are defined by

$$
\begin{aligned}
F(\boldsymbol{r}) & :=\int_{\mathcal{E}_{\mathcal{D}}} g_{\mathcal{D}} \boldsymbol{r} \cdot \boldsymbol{n} d \boldsymbol{x}+\int_{\mathcal{E}_{\mathcal{N}}} C_{22}\left(\boldsymbol{g}_{\mathcal{N}} \cdot \boldsymbol{n}\right)(\boldsymbol{r} \cdot \boldsymbol{n}) d s \\
G(v) & :=\int_{\Omega} f v d \boldsymbol{x}+\int_{\mathcal{E}_{\mathcal{D}}} C_{11} g_{\mathcal{D}} v d s+\int_{\mathcal{E}_{\mathcal{N}}} v \boldsymbol{g}_{\mathcal{N}} \cdot \boldsymbol{n} d s .
\end{aligned}
$$


Note that these two linear forms contain all the data of the problem. In particular, they contain both the Dirichlet and Neumann data, which is not the case for the classical mixed finite element methods.

Equations (2.6) and (2.7) can be rewritten in a more compact form as follows:

$$
\mathcal{A}\left(\boldsymbol{q}_{N}, u_{N} ; \boldsymbol{r}, v\right)=\mathcal{F}(\boldsymbol{r}, v),
$$

by setting

$$
\begin{aligned}
\mathcal{A}(\boldsymbol{q}, u ; \boldsymbol{r}, v) & :=a(\boldsymbol{q}, \boldsymbol{r})+b(u, \boldsymbol{r})-b(v, \boldsymbol{q})+c(u, v), \\
\mathcal{F}(\boldsymbol{r}, v) & :=F(\boldsymbol{r})+G(v) .
\end{aligned}
$$

We end this section by proving Proposition 2.1.

Proof of Proposition 2.1. Due to the linearity and finite dimensionality of the problem, it is enough to show that the only solution to the equations (2.6) and (2.7) with $f=0$, $g_{\mathcal{D}}=0$ and $\boldsymbol{g}_{\mathcal{N}}=0$ is $\boldsymbol{q}_{N}=0$ and $u_{N}=0$. Indeed, taking $\boldsymbol{r}=\boldsymbol{q}_{N}, v=u_{N}$ and adding the two equations, we get

$$
a\left(\boldsymbol{q}_{N}, \boldsymbol{q}_{N}\right)+c\left(u_{N}, u_{N}\right)=0,
$$

which implies $\boldsymbol{q}_{N}=0$, since $C_{22} \geq 0$, and $\llbracket u_{N} \rrbracket=0$ on $\mathcal{E}_{i}, u_{N}=0$ on $\mathcal{E}_{\mathcal{D}}$, since $C_{11}>0$. As a consequence, equation (2.6) becomes

$$
b\left(u_{N}, \boldsymbol{r}\right)=0 \quad \forall \boldsymbol{r} \in \boldsymbol{M}_{N} .
$$

Since, after integration by parts, the form $b(\cdot, \cdot)$ can be rewritten as

$$
b(u, \boldsymbol{r})=-\sum_{K \in \mathcal{T}} \int_{K} \nabla u \cdot \boldsymbol{r} d \boldsymbol{x}+\int_{\mathcal{E}_{i}}\left(\left\{\boldsymbol{r} \rrbracket-\boldsymbol{C}_{12} \llbracket \boldsymbol{r} \rrbracket\right) \cdot \llbracket u \rrbracket d s+\int_{\mathcal{E}_{\mathcal{D}}} u \boldsymbol{r} \cdot \boldsymbol{n} d s,\right.
$$

we get that

$$
b\left(u_{N}, \boldsymbol{r}\right)=\sum_{K \in \mathcal{T}} \int_{K} \nabla u_{N} \cdot \boldsymbol{r} d \boldsymbol{x}=0 \quad \forall \boldsymbol{r} \in \boldsymbol{M}_{N} .
$$

Hence, owing to (2.1), $\nabla u_{N}=0$ on every $K \in \mathcal{T}$ and since $u_{N}$ is a continuous function equal to zero on the Dirichlet boundary, we get that $u_{N} \equiv 0$. This completes the proof of Proposition 2.1.

2.3. A priori error estimates. In this section we state and discuss our a priori error bounds for the DG method. As pointed out at the beginning of this section, we restrict our analysis to domains $\Omega$ such that, for smooth data, the solution $u$ of problem (1.1) belongs to $H^{2}(\Omega)$. We also assume that when $f$ is in $\mathrm{L}^{2}(\Omega)$ and the boundary data are zero, we have the elliptic regularity result $\|u\|_{2} \leq C\|f\|_{0}$; see Grisvard [28] or [29].

We assume that every element $K$ of the triangulation $\mathcal{T}$ is affine equivalent, see [16, Section 2.3], to one of several reference elements in an arbitrary but fixed set; this allows us to use elements of various shapes with possibly curved boundaries. For each $K \in \mathcal{T}$, we denote by $h_{K}$ the diameter of $K$ and by $\rho_{K}$ the diameter of the biggest ball included in $K$; we set, as usual, $h:=\max _{K \in \mathcal{T}} h_{K}$. The triangulations we consider can 
have hanging nodes but have to be regular, that is, there exists a positive constant $\sigma$ such that

$$
\frac{h_{K}}{\rho_{K}} \leq \sigma, \quad \forall K \in \mathcal{T}
$$

see [16, Section 3.1]. Moreover, we let the maximum number of neighbors of a given element $\mathrm{K}$ be arbitrary but fixed. To formally state this property, we need to introduce the set $\left\langle K, K^{\prime}\right\rangle$ defined as follows:

$$
\left\langle K, K^{\prime}\right\rangle= \begin{cases}\emptyset & \text { if } \operatorname{meas}_{(d-1)}\left(\partial K \cap \partial K^{\prime}\right)=0, \\ \text { interior of } \partial K \cap \partial K^{\prime} & \text { otherwise. }\end{cases}
$$

Thus, we assume that there exists a positive constant $\delta<1$ such that, for each element $K \in \mathcal{T}$,

$$
\delta \leq \frac{h_{K^{\prime}}}{h_{K}} \leq \delta^{-1} \quad \forall K^{\prime}:\left\langle K, K^{\prime}\right\rangle \neq \emptyset .
$$

These three hypotheses allow for quite general triangulations and are not restrictive in practice.

The only assumptions we use for the local space $\mathcal{S}(K)$ are that it contains the space $\mathcal{P}^{k}(K)$ of polynomials of degree at most $k$ on $K$ and satisfies (2.1).

Next, we introduce a semi-norm that appears in a natural way in the analysis of these methods. We denote by $H^{k}(D), D$ being a domain in $\mathbb{R}^{d}$, the Sobolev spaces of integer orders, and by $\|\cdot\|_{k, D}$ and $|\cdot|_{k, D}$ the usual norms and semi-norms in $H^{k}(D)$ and $H^{k}(D)^{d}$; we omit the dependence on the domain in the norms whenever $D=\Omega$. We define $|(\boldsymbol{q}, u)|_{\mathcal{A}}^{2}:=\mathcal{A}(\boldsymbol{q}, u ; \boldsymbol{q}, u)$, that is,

$$
|(\boldsymbol{q}, u)|_{\mathcal{A}}^{2}=\|\boldsymbol{q}\|_{0}^{2}+\Theta^{2}(\boldsymbol{q}, u)
$$

where

$$
\Theta^{2}(\boldsymbol{q}, u):=\int_{\mathcal{E}_{i}} C_{22} \llbracket \boldsymbol{q} \rrbracket^{2} d s+\int_{\mathcal{E}_{\mathcal{N}}} C_{22}(\boldsymbol{q} \cdot \boldsymbol{n})^{2} d s+\int_{\mathcal{E}_{i}} C_{11} \llbracket u \rrbracket^{2} d s+\int_{\mathcal{E}_{\mathcal{D}}} C_{11} u^{2} d s .
$$

We assume that the stabilization coefficients $C_{11}$ and $C_{22}$ defining the numerical fluxes in (2.4) and (2.5) are defined as follows:

$$
\begin{aligned}
& C_{11}(\boldsymbol{x})= \begin{cases}\zeta \min \left\{h_{K^{+}}^{\alpha}, h_{K^{-}}^{\alpha}\right\} & \text { if } \boldsymbol{x} \in\left\langle K^{+}, K^{-}\right\rangle, \\
\zeta h_{K^{+}}^{\alpha} & \text { if } \boldsymbol{x} \in \partial K^{+} \cap \Gamma_{\mathcal{D}},\end{cases} \\
& C_{22}(\boldsymbol{x})= \begin{cases}\tau \min \left\{h_{K^{+}}^{\beta}, h_{K^{-}}^{\beta}\right\} & \text { if } \boldsymbol{x} \in\left\langle K^{+}, K^{-}\right\rangle, \\
\tau h_{K^{+}}^{\beta} & \text { if } \boldsymbol{x} \in \partial K^{+} \cap \Gamma_{\mathcal{N}},\end{cases}
\end{aligned}
$$

with $\zeta>0, \tau \geq 0,-1 \leq \alpha \leq 0 \leq \beta \leq 1$ independent of the mesh-size and $\left|\boldsymbol{C}_{12}\right|$ of order one. Our main result will be written in terms of the parameters $\mu^{\star}$ and $\mu_{\star}$ defined as follows:

$$
\mu^{\star}=\max \{-\alpha, \widehat{\beta}\}, \quad \mu_{\star}=\min \{-\alpha, \widehat{\beta}\},
$$

where $\widehat{\beta}=1$ if $\tau=0$ and $\widehat{\beta}=\beta$ otherwise. 
We are now ready to state our main result.

THEOREM 2.2. Let $(\boldsymbol{q}, u)$ be the solution of (1.2)-(1.5) and let $\left(\boldsymbol{q}_{N}, u_{N}\right)$ be the approximate solution given by the DG method (2.2) and (2.3). We assume the hypotheses on the local spaces and on the form of the stabilization parameters described above. The triangulations are assumed to satisfy the hypothesis (2.11); if $\alpha \neq 0$ or $\beta \neq 0$, we also assume that hypothesis (2.12) is satisfied. Then we have that, for $(\boldsymbol{q}, u) \in H^{s+1}(\Omega)^{d} \times H^{s+2}(\Omega)$ with $s \geq 0$,

$$
\left\|u-u_{N}\right\|_{0}+h^{D}\left|\left(\boldsymbol{q}-\boldsymbol{q}_{N}, u-u_{N}\right)\right|_{\mathcal{A}} \leq C h^{P+D}\|u\|_{s+2},
$$

where $C$ solely depends on $\sigma, \delta$ (not when $\alpha=\beta=0$ ), $\zeta, \tau, k$, and $d$; and

$$
P=\min \left\{s+\frac{1}{2}\left(1+\mu_{\star}\right), k+\frac{1}{2}\left(1-\mu^{\star}\right)\right\}, \quad D=\frac{1}{2}\left(1+\mu_{\star}\right) \quad \text { if } k \geq 1 .
$$

When $k=0$, we have $P=D=\frac{1}{2}\left(1-\mu^{\star}\right)$.

Let us briefly discuss the above result:

- We begin by noting that the orders of convergence depend on the size of the stabilization parameters $C_{11}$ and $C_{22}$ only through the quantities $\mu^{\star}$ and $\mu_{\star}$. This fact has several important consequences:

o The same orders of convergence are obtained with either $C_{22}=0$ or $C_{22}$ of order $h$. This means that there is no loss in the orders of convergence if instead of penalizing the jumps of the normal component of $\boldsymbol{q}_{N}$ with a $C_{22}$ or order $h$, no penalization at all (the LDG method) is used.

- The same orders of convergence are obtained with either the LDG method $\left(C_{22}=0\right)$ with $C_{11}$ of order one or $C_{11}$ of order $h^{-1}$ and $C_{22}$ of order one.

- In general, the same orders of convergence are obtained by taking $(\alpha, \beta)=$ $(-a, b)$ or by taking $(\alpha, \beta)=(-b, a)$.

- The most remarkable cases occur when $-\alpha, \beta \in\{0,1\}$ since it is for those values that $\mu^{\star}$ and $\mu_{\star}$ achieve their maximum and minimum. The corresponding orders of convergence are displayed in Table 2.1 for $k \geq 1$.

TABLE 2.1

\begin{tabular}{|c|c|c|c|}
\hline$C_{22}$ & $C_{11}$ & $\left|\left(\boldsymbol{q}-\boldsymbol{q}_{N}, u-u_{N}\right)\right|_{\mathcal{A}}$ & $\left\|u-u_{N}\right\|_{0}$ \\
\hline $\begin{array}{l}0, \mathcal{O}(h) \\
0, \mathcal{O}(h)\end{array}$ & $\begin{array}{c}\mathcal{O}(1) \\
\mathcal{O}(1 / h)\end{array}$ & $\begin{array}{c}\min \{s+1 / 2, k\} \\
\min \{s+1, k\}\end{array}$ & $\begin{array}{c}\min \{s+1 / 2, k\}+1 / 2 \\
\min \{s+1, k\}+1\end{array}$ \\
\hline $\begin{array}{l}\mathcal{O}(1) \\
\mathcal{O}(1)\end{array}$ & $\begin{array}{c}\mathcal{O}(1) \\
\mathcal{O}(1 / h)\end{array}$ & $\begin{array}{l}\min \{s, k\}+1 / 2 \\
\min \{s+1 / 2, k\}\end{array}$ & $\begin{array}{c}\min \{s, k\}+1 \\
\min \{s+1 / 2, k\}+1 / 2\end{array}$ \\
\hline
\end{tabular}

Orders of convergence for $u \in H^{s+2}(\Omega)$ for $s \geq 0$ and $k \geq 1$.

- In the case $1 \leq k \leq s$, that is, when the degree of the polynomial approximation is less than needed to fit the smoothness of the exact solution, we see in Table 2.2 that the best orders of convergence for $\left|\left(\boldsymbol{q}-\boldsymbol{q}_{N}, u-u_{N}\right)\right|_{\mathcal{A}}$ and $\left\|u-u_{N}\right\|_{0}, k+1 / 2$ 
and $k+1$, respectively, are obtained for both $C_{11}$ and $C_{22}$ of order one. When $C_{22}$ is taken to be of order $h$ or equal to zero, the stability of the method is weakened and, as a consequence, a loss in the orders of convergence of $1 / 2$ takes place. If now $C_{11}$ is taken to be of order $h^{-1}$, the full order of convergence in the error of the potential is recovered. The numerical experiments of section 5 show that these orders of convergence are actually achieved. However, the expected loss in the orders of convergence when $C_{11}$ is taken of order one is not observed, which shows that in practice the LDG method is essentially insensitive to the size of the stabilization parameter $C_{11}$.

- The influence of the choice of the coefficients $\boldsymbol{C}_{12}$ on the accuracy has not been explored in this paper; we only assume those to be of order one. In [22] it is shown that the LDG method, with a suitable choice of the coefficients $\boldsymbol{C}_{12}$, still gives the orders of convergence of $k+1 / 2$ and $k+1$ for $\left|\left(\boldsymbol{q}-\boldsymbol{q}_{N}, u-u_{N}\right)\right|_{\mathcal{A}}$ and $\left\|u-u_{N}\right\|_{0}$, respectively, if Cartesian grids and tensor product polynomials of degree $k$ in each variable are used.

TABLE 2.2

Orders of convergence for $u \in H^{s+2}(\Omega)$ for $s \geq k$ and $k \geq 1$.

\begin{tabular}{cc|c|c}
\hline$C_{22}$ & $C_{11}$ & $\left|\left(\boldsymbol{q}-\boldsymbol{q}_{N}, u-u_{N}\right)\right|_{\mathcal{A}}$ & $\left\|u-u_{N}\right\|_{0}$ \\
\hline $0, \mathcal{O}(h)$ & $\mathcal{O}(1)$ & $k$ & $k+1 / 2$ \\
$0, \mathcal{O}(h)$ & $\mathcal{O}(1 / h)$ & $k$ & $k+1$ \\
\hline $\mathcal{O}(1)$ & $\mathcal{O}(1)$ & $k+1 / 2$ & $k+1$ \\
$\mathcal{O}(1)$ & $\mathcal{O}(1 / h)$ & $k$ & $k+1$ \\
\hline
\end{tabular}

- For the case $k \geq s+1$, that is, when the degree of the polynomial approximation is more than needed to fit the smoothness of the exact solution, we see in Table 2.3 that the LDG method performs at least as well as all the other methods; it performs better if $C_{11}$ is of order $h^{-1}$.

TABLE 2.3

Orders of convergence for $u \in H^{s+2}(\Omega)$ for $s \geq 0$ and $k \geq s+1$.

\begin{tabular}{cc|c|c}
\hline$C_{22}$ & $C_{11}$ & $\left|\left(\boldsymbol{q}-\boldsymbol{q}_{N}, u-u_{N}\right)\right|_{\mathcal{A}}$ & $\left\|u-u_{N}\right\|_{0}$ \\
\hline & & $s+1 / 2$ & $s+1$ \\
$0, \mathcal{O}(h)$ & $\mathcal{O}(1)$ & $s+1$ & $s+2$ \\
$0, \mathcal{O}(h)$ & $\mathcal{O}(1 / h)$ & & \\
\hline & & $s+1 / 2$ & $s+1$ \\
$\mathcal{O}(1)$ & $\mathcal{O}(1)$ & $s+1 / 2$ & $s+1$ \\
$\mathcal{O}(1)$ & $\mathcal{O}(1 / h)$ & & \\
\hline
\end{tabular}


- In the case $k=0$, the DG method converges provided $C_{22} \neq 0$; in particular, for constant coefficients $C_{11}$ and $C_{22}$, we obtain estimates of order one for $\left\|u-u_{N}\right\|_{0}$, and $1 / 2$ for $\left|\left(\boldsymbol{q}-\boldsymbol{q}_{N}, u-u_{N}\right)\right|_{\mathcal{A}}$. This is one of the few finite element methods for secondorder elliptic problems that actually converges for piecewise-constant approximations. When $C_{22}=0$, that is, for the LDG method, our numerical results, which we do not report in this paper, show that there is no positive order of convergence in this case, as predicted by Theorem 2.2 .

- Finally, let us point out that the hypothesis (2.12) is not necessary when $\alpha=\beta=0$.

2.4. The idea of the proof. The proof of Theorem 2.2 will be carried out in section 3. The purpose of this section is to display as clearly as possible the basic ingredients and the main steps of our error analysis. As usual, we express the error $\left(\boldsymbol{e} \boldsymbol{q}, e_{u}\right)=\left(\boldsymbol{q}-\boldsymbol{q}_{N}, u-u_{N}\right)$ as the following sum:

$$
\left(e_{\boldsymbol{q}}, e_{u}\right)=(\boldsymbol{q}-\Pi \boldsymbol{q}, u-\Pi u)+\left(\boldsymbol{\Pi} e_{\boldsymbol{q}}, \Pi e_{u}\right),
$$

where $\Pi$ and $\Pi$ are projections from $\boldsymbol{M}$ and $V$ onto the finite element spaces $\boldsymbol{M}_{N}$ and $V_{N}$, respectively.

a. The basic ingredients. The basic ingredients of our error analysis are two. The first one is, as it is classical in finite element error analysis, the so-called Galerkin orthogonality property, namely,

$$
\mathcal{A}\left(\boldsymbol{e}_{\boldsymbol{q}}, e_{u} ; \boldsymbol{r}, v\right)=0 \quad \forall(\boldsymbol{r}, v) \in \boldsymbol{M}_{N} \times V_{N} .
$$

This property is a straightforward consequence of the consistency of the numerical fluxes.

The second ingredient is a couple of inequalities that reflect the approximation properties of the projections $\Pi$ and $\Pi$, namely,

$$
|\mathcal{A}(\boldsymbol{q}-\Pi \boldsymbol{q}, u-\Pi u ; \boldsymbol{\Phi}-\Pi \mathbf{\Phi}, \varphi-\Pi \varphi)| \leq K_{\mathcal{A}}(\boldsymbol{q}, u ; \boldsymbol{\Phi}, \varphi)
$$

for any $(\boldsymbol{q}, u),(\boldsymbol{\Phi}, \varphi) \in \boldsymbol{M} \times V$, and

$$
|\mathcal{A}(\boldsymbol{r}, v ; \boldsymbol{q}-\Pi \boldsymbol{q}, u-\Pi u)| \leq|(\boldsymbol{r}, v)|_{\mathcal{A}} K_{\mathcal{B}}(\boldsymbol{q}, u)
$$

for any $(\boldsymbol{r}, v) \in \boldsymbol{M}_{N} \times V_{N}$ and $(\boldsymbol{q}, u) \in H^{1}(\Omega)^{d} \times H^{2}(\Omega)$.

As we show next, all the error estimates we are interested in can be obtained solely in terms of functionals $K_{\mathcal{A}}$ and $K_{\mathcal{B}}$.

b. The estimate of the error in the $\mathcal{A}$-semi-norm. We have the following result. LEMma 2.3. We have

$$
\left|\left(\boldsymbol{e}_{\boldsymbol{q}}, e_{u}\right)\right|_{\mathcal{A}} \leq K_{\mathcal{A}}^{1 / 2}(\boldsymbol{q}, u ; \boldsymbol{q}, u)+K_{\mathcal{B}}(\boldsymbol{q}, u)
$$

Proof. $|(\cdot, \cdot)|_{\mathcal{A}}$ is a semi-norm and, hence,

$$
\left|\left(e_{\boldsymbol{q}}, e_{u}\right)\right|_{\mathcal{A}} \leq|(\boldsymbol{q}-\Pi \boldsymbol{\eta}, u-\Pi u)|_{\mathcal{A}}+\left|\left(\boldsymbol{\Pi} e_{\boldsymbol{q}}, \Pi e_{u}\right)\right|_{\mathcal{A}} .
$$

Since

$$
\begin{aligned}
\left|\left(\Pi e_{\boldsymbol{q}}, \Pi e_{u}\right)\right|_{\mathcal{A}}^{2} & =\mathcal{A}\left(\Pi e_{\boldsymbol{q}}, \Pi e_{u} ; \Pi e_{\boldsymbol{q}}, \Pi e_{u}\right) \\
& =\mathcal{A}\left(\Pi \boldsymbol{q}-\boldsymbol{q}, \Pi u-u ; \Pi e_{\boldsymbol{q}}, \Pi e_{u}\right) \quad \text { by Galerkin orthogonality (2.17), } \\
& =\mathcal{A}\left(-\Pi e_{\boldsymbol{q}}, \Pi e_{u} ; \boldsymbol{q}-\Pi \boldsymbol{q}, \Pi u-u\right) \quad \text { by the definition of } \mathcal{A},(2.9), \\
& \leq\left|\left(\Pi e_{\boldsymbol{q}}, \Pi e_{u}\right)\right|_{\mathcal{A}} K_{\mathcal{B}}(\boldsymbol{q}, u) \quad \text { by assumption }(2.19),
\end{aligned}
$$


we have that

$$
\left|\left(\Pi e_{\boldsymbol{q}}, \Pi e_{u}\right)\right|_{\mathcal{A}} \leq K_{\mathcal{B}}(\boldsymbol{q}, u)
$$

and so,

$$
\left|\left(\boldsymbol{e} \boldsymbol{q}, e_{u}\right)\right|_{\mathcal{A}} \leq|(\boldsymbol{q}-\Pi \boldsymbol{q}, u-\Pi u)|_{\mathcal{A}}+K_{\mathcal{B}}(\boldsymbol{q}, u) .
$$

The estimate now follows from a simple application of the assumption (2.18). This completes the proof. $\square$

c. Estimate of the error in $u$ in non-positive order norms. To obtain an estimate of $\left\|e_{u}\right\|_{-t, D}$, where $t$ is a natural number and $D$ is a sub-domain of $\Omega$, we only have to obtain an estimate of the error in the approximation of the linear functional $\Lambda(u)=(\lambda, u)$, where $(\cdot, \cdot)$ denotes the $L^{2}$-inner product, by $\Lambda\left(u_{N}\right)$ since

$$
\left\|e_{u}\right\|_{-t, D}=\sup _{\lambda \in \mathcal{C}_{0}^{\infty}(D)} \frac{\Lambda\left(e_{u}\right)}{\|\lambda\|_{t, D}}
$$

In this paper, we are only interested in the case $t=0$, but we give here the general argument to stress the fact that it is essentially the same for all natural numbers $t$. Error estimates in negative order norms are very important, as we point out in section 4 of this paper.

To obtain our estimate, we need to introduce the solution $\varphi$ of the so-called adjoint problem, namely,

$$
\begin{aligned}
-\Delta \varphi=\lambda & & \text { in } \Omega, \\
\varphi=0 & & \text { on } \Gamma_{\mathcal{D}}, \\
\frac{\partial \varphi}{\partial n}=0 & & \text { on } \Gamma_{\mathcal{N}} .
\end{aligned}
$$

Lemma 2.4. Let $t$ be a natural number. Then, we have

$$
\left\|e_{u}\right\|_{-t, D} \leq \sup _{\lambda \in \mathcal{C}_{0}^{\infty}(D)} \frac{K_{\mathcal{A}}(\boldsymbol{q}, u ; \boldsymbol{\Phi}, \varphi)}{\|\lambda\|_{t, D}}+K_{\mathcal{B}}(\boldsymbol{q}, u) \sup _{\lambda \in \mathcal{C}_{0}^{\infty}(D)} \frac{K_{\mathcal{B}}(\mathbf{\Phi}, \varphi)}{\|\lambda\|_{t, D}}
$$

with $\varphi$ denoting the solution of (2.21)-(2.23) and $\Phi=-\nabla \varphi$.

Proof. Since $\varphi$ is the solution of the adjoint equation, it is easy to verify that if we set $\mathbf{\Phi}=-\nabla \varphi$, we have

$$
\mathcal{A}(-\boldsymbol{\Phi}, \varphi ;-\boldsymbol{s}, w)=\Lambda(w),
$$

for all $(s, w) \in \boldsymbol{M} \times V$; indeed, note that problem (1.1) can be rewritten as in (2.8). Taking $(\boldsymbol{s}, w)=\left(\boldsymbol{e}_{\boldsymbol{q}}, e_{u}\right)$, we get

$$
\begin{array}{rlr}
\Lambda\left(e_{u}\right) & =\mathcal{A}\left(\boldsymbol{e}_{\boldsymbol{q}}, e_{u} ; \boldsymbol{\Phi}, \varphi\right) & \text { by the definition of } \mathcal{A},(2.9), \\
& =\mathcal{A}\left(\boldsymbol{e}_{\boldsymbol{q}}, e_{u} ; \boldsymbol{\Phi}-\Pi \mathbf{\Phi}, \varphi-\Pi \varphi\right) & \text { by Galerkin orthogonality }(2.17), \\
& =\mathcal{A}\left(\Pi \boldsymbol{e}_{\boldsymbol{q}}, \Pi e_{u} ; \boldsymbol{\Phi}-\Pi \boldsymbol{\Phi}, \varphi-\Pi \varphi\right)+\mathcal{A}(\boldsymbol{q}-\Pi \boldsymbol{q}, u-\Pi u ; \boldsymbol{\Phi}-\Pi \mathbf{\Phi}, \varphi-\Pi \varphi) .
\end{array}
$$

Since $\left(\Pi e \boldsymbol{q}, \Pi e_{u}\right) \in \boldsymbol{M}_{N} \times V_{N}$, by the assumption (2.19) and the estimate (2.20), we obtain

$$
\left|\mathcal{A}\left(\Pi e_{\boldsymbol{q}}, \Pi e_{u} ; \boldsymbol{\Phi}-\Pi \mathbf{\Phi}, \varphi-\Pi \varphi\right)\right| \leq K_{\mathcal{B}}(\boldsymbol{q}, u) K_{\mathcal{B}}(\boldsymbol{\Phi}, \varphi)
$$


and hence,

$$
\begin{aligned}
\left|\Lambda\left(e_{u}\right)\right| \leq & K_{\mathcal{B}}(\boldsymbol{q}, u) K_{\mathcal{B}}(\boldsymbol{\Phi}, \varphi) \\
& +|\mathcal{A}(\boldsymbol{q}-\Pi \boldsymbol{\Pi}, u-\Pi u ; \boldsymbol{\Phi}-\Pi \boldsymbol{\Phi}, \varphi-\Pi \varphi)| .
\end{aligned}
$$

The estimate now follows from a simple application of assumption (2.18), and from the definition of a non-positive order norm. This completes the proof. $\square$

d. Conclusion. Thus, in order to prove our a priori estimates, all we need to do is to obtain the functionals $K_{\mathcal{A}}$ and $K_{\mathcal{B}}$; this will be carried out in the next section. Then, Theorem 2.2 will immediately follow after a simple application of Lemmas 2.3 and 2.4.

3. Proofs. In this section, we prove our main results. We proceed as follows. First, we obtain the functional $K_{\mathcal{A}}$ for general projection operators $\Pi$ and $\Pi$. To obtain the functional $K_{\mathcal{B}}$, the projections $\Pi$ and $\Pi$ are taken to be the standard $\mathrm{L}^{2}$ projections, just as done by Cockburn and Shu [25] in their study of the LDG method for transient convection-diffusion problems.

3.1. Preliminaries. The following two lemmas contain all the information we actually use about our finite elements. The first one is a standard approximation result for any linear continuous operator $\Pi$ from $H^{r+1}(K)$ onto $\mathcal{S}(K)$ satisfying $\Pi w=w$ for any $w \in \mathcal{P}^{k}(K)$; it can be easily obtained by using the techniques of [16]. The second one is a standard inverse inequality.

Lemma 3.1. Let $w \in H^{r+1}(K), r \geq 0$. Let $\Pi$ be a linear continuous operator from $H^{r+1}(K)$ onto $\mathcal{S}(K)$ such that $\Pi w=w$ for all $w \in \mathcal{P}^{k}(K)$. Then for $m$ integer, $0 \leq m \leq r+1$, we have

$$
\begin{aligned}
& |w-\Pi w|_{m, K} \leq C h_{K}^{\min \{r, k\}+1-m}\|w\|_{r+1, K}, \\
& \|w-\Pi w\|_{0, \partial K} \leq C h_{K}^{\min \{r, k\}+\frac{1}{2}}\|w\|_{r+1, K},
\end{aligned}
$$

for some constant $C$ that solely depends on $\sigma$ in inequality (2.11), $k, d$ and $r$.

Lemma 3.2. There exists a positive constant $C_{\mathrm{inv}}$ that solely depends on $\sigma$ in inequality (2.11), $k$ and $d$, such that for all $s \in \mathcal{S}(K)^{d}$ we have

$$
\|s\|_{0, \partial K} \leq C_{\mathrm{inv}} h_{K}^{-\frac{1}{2}}\|s\|_{0, K},
$$

for all $K \in \mathcal{T}$.

We are now ready to prove our main result.

3.2. The functional $K_{\mathcal{A}}$. In this subsection we determine the functional $K_{\mathcal{A}}$ in (2.18), up to a multiplicative constant independent of the mesh-size. We start by giving an expression for $K_{\mathcal{A}}$ which is valid for coefficients $C_{11}$ and $C_{22}$ that vary from face to face, for $k=0$ and for any regularity of the solution. Then we write $K_{\mathcal{A}}$ for the particular choice (2.15), (2.16) of $C_{11}$ and $C_{22}$ in Theorem 2.2.

Let $\Pi$ and $\Pi$ be arbitrary projections onto $V_{N}$ and $\boldsymbol{M}_{N}$, respectively, satisfying (component-wisely) the assumptions in Lemma 3.1.

Lemma 3.3. Assume $(\boldsymbol{q}, u) \in H^{s+1}(\Omega)^{2} \times H^{s+2}(\Omega)$ and $(\boldsymbol{\Phi}, \varphi) \in H^{t+1}(\Omega)^{2} \times$ $H^{t+2}(\Omega), s, t \geq 0$. Then the approximation property (2.18) holds true with

$$
K_{\mathcal{A}}(\boldsymbol{q}, u ; \boldsymbol{\Phi}, \varphi)=\sum_{i=1}^{5} S_{i}(\boldsymbol{q}, u ; \boldsymbol{\Phi}, \varphi),
$$


where

$\begin{aligned} S_{1} & :=C\left(\sum_{K \in \mathcal{T}} h_{K}^{2 \min \{s, k\}+2}\|\boldsymbol{q}\|_{s+1, K}^{2}\right)^{\frac{1}{2}}\left(\sum_{K \in \mathcal{T}} h_{K}^{2 \min \{t, k\}+2}\|\boldsymbol{\Phi}\|_{t+1, K}^{2}\right)^{\frac{1}{2}}, \\ S_{2} & :=C\left(\sum_{K \in \mathcal{T}} C_{22}^{\partial K} h_{K}^{2 \min \{s, k\}+1}\|\boldsymbol{q}\|_{s+1, K}^{2}\right)^{\frac{1}{2}}\left(\sum_{K \in \mathcal{T}} C_{22}^{\partial K} h_{K}^{2 \min \{t, k\}+1}\|\boldsymbol{\Phi}\|_{t+1, K}^{2}\right)^{\frac{1}{2}}, \\ S_{3} & :=C\left(\sum_{K \in \mathcal{T}} h_{K}^{2 \min \{s+1, k\}}\|u\|_{s+2, K}^{2}\right)^{\frac{1}{2}}\left(\sum_{K \in \mathcal{T}} \widehat{h}_{K}^{2 \min \{t, k\}+2}\|\boldsymbol{\Phi}\|_{t+1, K}^{2}\right)^{\frac{1}{2}}, \\ S_{4} & :=C\left(\sum_{K \in \mathcal{T}} \widehat{h}_{K}^{2 \min \{s, k\}+2}\|\boldsymbol{q}\|_{s+1, K}^{2}\right)^{\frac{1}{2}}\left(\sum_{K \in \mathcal{T}} h_{K}^{2 \min \{t+1, k\}}\|\varphi\|_{t+2, K}^{2}\right)^{\frac{1}{2}}, \\ S_{5} & :=C\left(\sum_{K \in \mathcal{T}} C_{11}^{\partial K} h_{K}^{2 \min \{s+1, k\}+1}\|u\|_{s+2, K}^{2}\right)^{\frac{1}{2}}\left(\sum_{K \in \mathcal{T}} C_{11}^{\partial K} h_{K}^{2 \min \{t+1, k\}+1}\|\varphi\|_{t+2, K}^{2}\right)^{\frac{1}{2}},\end{aligned}$

$\widehat{h}_{K}:=\sup \left\{h_{K^{\prime}}:\left\langle K, K^{\prime}\right\rangle \neq \emptyset\right\}, C_{i i}^{\partial K}:=\sup \left\{C_{i i}(\boldsymbol{x}): \boldsymbol{x} \in \partial K\right\}, i=1,2$. The positive constant $C$ is independent of the mesh-size but depends on the approximation constants in Lemma 3.1 and on the coefficients $\boldsymbol{C}_{12}$.

Furthermore, in the case where $(\boldsymbol{\Phi}, \varphi)=(\boldsymbol{q}, u)$, we have

$$
K_{\mathcal{A}}(\boldsymbol{q}, u ; \boldsymbol{q}, u)=S_{1}(\boldsymbol{q}, u ; \boldsymbol{q}, u)+S_{2}(\boldsymbol{q}, u ; \boldsymbol{q}, u)+S_{5}(\boldsymbol{q}, u ; \boldsymbol{q}, u) .
$$

Proof. We set, for convenience, $\boldsymbol{\xi}_{\boldsymbol{q}}:=\boldsymbol{q}-\boldsymbol{\Pi} \boldsymbol{q}, \xi_{u}:=u-\Pi u, \boldsymbol{\xi}_{\boldsymbol{\Phi}}:=\boldsymbol{\Phi}-\boldsymbol{\Pi} \boldsymbol{\Phi}$, $\xi_{\varphi}:=\varphi-\Pi \varphi$. We start by writing

$$
\mathcal{A}\left(\boldsymbol{\xi}_{\boldsymbol{q}}, \xi_{u} ; \boldsymbol{\xi}_{\boldsymbol{\Phi}}, \xi_{\varphi}\right)=a\left(\boldsymbol{\xi}_{\boldsymbol{q}}, \boldsymbol{\xi}_{\boldsymbol{\Phi}}\right)+b\left(\xi_{u}, \boldsymbol{\xi}_{\boldsymbol{\Phi}}\right)-b\left(\xi_{\varphi}, \boldsymbol{\xi}_{\boldsymbol{q}}\right)+c\left(\xi_{u}, \xi_{\varphi}\right),
$$

and then proceed by estimating each of the forms on the right-hand side separately. The form $a(\cdot, \cdot)$ can be written as

$$
\begin{aligned}
a\left(\boldsymbol{\xi}_{\boldsymbol{q}}, \boldsymbol{\xi}_{\boldsymbol{\Phi}}\right)= & \sum_{K \in \mathcal{T}}\left(\int_{K} \boldsymbol{\xi}_{\boldsymbol{q}} \cdot \boldsymbol{\xi}_{\boldsymbol{\Phi}} d \boldsymbol{x}+\int_{\partial K \cap \Gamma_{\mathcal{N}}} C_{22}\left(\boldsymbol{\xi}_{\boldsymbol{q}} \cdot \boldsymbol{n}\right)\left(\boldsymbol{\xi}_{\boldsymbol{\Phi}} \cdot \boldsymbol{n}\right) d s\right. \\
& \left.+\int_{\partial K \backslash \partial \Omega} C_{22}\left(\boldsymbol{\xi}_{\boldsymbol{q}} \cdot \boldsymbol{n}\right)\left(\boldsymbol{\xi}_{\boldsymbol{\Phi}} \cdot \boldsymbol{n}\right) d s+\int_{\partial K \backslash \partial \Omega} C_{22}\left(\boldsymbol{\xi}_{\boldsymbol{q}}^{\text {out }} \cdot \boldsymbol{n}^{\text {out }}\right)\left(\boldsymbol{\xi}_{\boldsymbol{\Phi}} \cdot \boldsymbol{n}\right) d s\right)
\end{aligned}
$$

where the superscript 'out' denotes quantities taken on $\partial K \backslash \partial \Omega$ from outside $K$. By repeated applications of the Cauchy-Schwarz's inequality, we obtain that $\left|a\left(\boldsymbol{\xi}_{\boldsymbol{q}}, \boldsymbol{\xi}_{\boldsymbol{\Phi}}\right)\right|$ is bounded by

$$
\begin{aligned}
& \sum_{K \in \mathcal{T}}\left(\left\|\boldsymbol{\xi}_{\boldsymbol{q}}\right\|_{0, K}\left\|\boldsymbol{\xi}_{\boldsymbol{\Phi}}\right\|_{0, K}+\left\|C_{22}^{\frac{1}{2}} \boldsymbol{\xi}_{\boldsymbol{q}} \cdot \boldsymbol{n}\right\|_{0, \partial K \cap \Gamma_{\mathcal{N}}}\left\|C_{22}^{\frac{1}{2}} \boldsymbol{\xi}_{\boldsymbol{\Phi}} \cdot \boldsymbol{n}\right\|_{0, \partial K \cap \Gamma_{\mathcal{N}}}\right. \\
& \left.\quad+\left(\left\|C_{22}^{\frac{1}{2}} \boldsymbol{\xi}_{\boldsymbol{q}} \cdot \boldsymbol{n}\right\|_{0, \partial K \backslash \partial \Omega}+\left\|C_{22}^{\frac{1}{2}} \boldsymbol{\xi}_{\boldsymbol{q}}^{\text {out }} \cdot \boldsymbol{n}^{\text {out }}\right\|_{0, \partial K \backslash \partial \Omega}\right)\left\|C_{22}^{\frac{1}{2}} \boldsymbol{\xi}_{\boldsymbol{\Phi}} \cdot \boldsymbol{n}\right\|_{0, \partial K \backslash \partial \Omega}\right) \\
& \leq\left(\sum_{K \in \mathcal{T}}\left\|\boldsymbol{\xi}_{\boldsymbol{q}}\right\|_{0, K}^{2}\right)^{\frac{1}{2}}\left(\sum_{K \in \mathcal{T}}\left\|\boldsymbol{\xi}_{\boldsymbol{\Phi}}\right\|_{0, K}^{2}\right)^{\frac{1}{2}}
\end{aligned}
$$




$$
+2\left(\sum_{K \in \mathcal{T}} C_{22}^{\partial K}\left\|\boldsymbol{\xi}_{\boldsymbol{q}} \cdot \boldsymbol{n}\right\|_{0, \partial K}^{2}\right)^{\frac{1}{2}}\left(\sum_{K \in \mathcal{T}} C_{22}^{\partial K}\left\|\boldsymbol{\xi}_{\boldsymbol{\Phi}} \cdot \boldsymbol{n}\right\|_{0, \partial K}^{2}\right)^{\frac{1}{2}}
$$

Now, a straightforward application of Lemma 3.1 yields

$$
\left|a\left(\boldsymbol{\xi}_{\boldsymbol{q}}, \boldsymbol{\xi}_{\boldsymbol{\Phi}}\right)\right| \leq S_{1}(\boldsymbol{q}, u ; \boldsymbol{\Phi}, \varphi)+S_{2}(\boldsymbol{q}, u ; \boldsymbol{\Phi}, \varphi) .
$$

To deal with the second term, we first note that

$$
\begin{aligned}
b\left(\xi_{u}, \boldsymbol{\xi}_{\boldsymbol{\Phi}}\right)= & -\sum_{K \in \mathcal{T}}\left(\int_{K} \nabla \xi_{u} \cdot \boldsymbol{\xi}_{\boldsymbol{\Phi}} d \boldsymbol{x}+\int_{\partial K \cap \Gamma_{\mathcal{D}}} \xi_{u}\left(\boldsymbol{\xi}_{\boldsymbol{\Phi}} \cdot \boldsymbol{n}\right) d s\right. \\
& +\int_{\partial K \backslash \partial \Omega} \xi_{u}\left(\left\{\left\{\boldsymbol{\xi}_{\boldsymbol{\Phi}}\right\}-\boldsymbol{C}_{12} \llbracket \boldsymbol{\xi}_{\boldsymbol{\Phi}} \rrbracket\right) d s\right)
\end{aligned}
$$

and obtain, after repeated applications of the Cauchy-Schwarz's inequality with suitably chosen weights, that $\left|b\left(\xi_{u}, \boldsymbol{\xi}_{\boldsymbol{\Phi}}\right)\right|$ is bounded by

$$
\begin{aligned}
& \sum_{K \in \mathcal{T}}\left(\left|\xi_{u}\right|_{1, K}\left\|\boldsymbol{\xi}_{\boldsymbol{\Phi}}\right\|_{0, K}+\left\|h_{K}^{-\frac{1}{2}} \xi_{u}\right\|_{0, \partial K \cap \Gamma_{\mathcal{D}}}\left\|h_{K}^{\frac{1}{2}} \boldsymbol{\xi}_{\boldsymbol{\Phi}} \cdot \boldsymbol{n}\right\|_{0, \partial K \cap \Gamma_{\mathcal{D}}}\right. \\
& \left.\quad+\left\|h_{K}^{-\frac{1}{2}} \xi_{u}\right\|_{0, \partial K \backslash \partial \Omega}\left\|h_{K}^{\frac{1}{2}}\left(\frac{1}{2} \boldsymbol{\xi}_{\boldsymbol{\Phi}}+\frac{1}{2} \boldsymbol{\xi}_{\boldsymbol{\Phi}}^{\text {out }}-\boldsymbol{C}_{12} \cdot \boldsymbol{n} \boldsymbol{\xi}_{\boldsymbol{\Phi}}-\boldsymbol{C}_{12} \cdot \boldsymbol{n}^{\text {out }} \boldsymbol{\xi}_{\boldsymbol{\Phi}}^{\text {out }}\right)\right\|_{0, \partial K \backslash \partial \Omega}\right) \\
& \leq\left(\sum_{K \in \mathcal{T}}\left(\left|\xi_{u}\right|_{1, K}^{2}+\frac{1}{h_{K}}\left\|\xi_{u}\right\|_{0, \partial K}^{2}\right)\right)^{\frac{1}{2}}\left(\sum_{K \in \mathcal{T}}\left(\left\|\boldsymbol{\xi}_{\boldsymbol{\Phi}}\right\|_{0, K}^{2}+4 \widehat{h}_{K}\left\|\left(\frac{1}{2}-\boldsymbol{C}_{12} \cdot \boldsymbol{n}\right) \boldsymbol{\xi}_{\boldsymbol{\Phi}}\right\|_{0, \partial K}^{2}\right)\right)^{\frac{1}{2}} \\
& \leq C\left(\sum_{K \in \mathcal{T}}\left(\left|\xi_{u}\right|_{1, K}^{2}+\frac{1}{h_{K}}\left\|\xi_{u}\right\|_{0, \partial K}^{2}\right)\right)^{\frac{1}{2}}\left(\sum_{K \in \mathcal{T}}\left(\left\|\boldsymbol{\xi}_{\boldsymbol{\Phi}}\right\|_{0, K}^{2}+\widehat{h}_{K}\left\|\boldsymbol{\xi}_{\boldsymbol{\Phi}}\right\|_{0, \partial K}^{2}\right)\right)^{\frac{1}{2}},
\end{aligned}
$$

where $C=1+2 \sup \left\{\left|\boldsymbol{C}_{12}(\boldsymbol{x}) \cdot \boldsymbol{n}\right|: \boldsymbol{x} \in \partial K\right\}$ and $\widehat{h}_{K}=\sup \left\{h_{K^{\prime}}:\left\langle K, K^{\prime}\right\rangle \neq \emptyset\right\}$. Once again, a straightforward application of Lemma 3.1 gives that

$$
\left|b\left(\xi_{u}, \boldsymbol{\xi}_{\boldsymbol{\Phi}}\right)\right| \leq S_{3}(\boldsymbol{q}, u ; \boldsymbol{\Phi}, \varphi) .
$$

For the third term, we use the same arguments to get

$$
\left|b\left(\xi_{\varphi}, \boldsymbol{\xi}_{\boldsymbol{q}}\right)\right| \leq S_{4}(\boldsymbol{q}, u ; \boldsymbol{\Phi}, \varphi) .
$$

Finally, proceeding as above, we get

$$
\begin{aligned}
\left|c\left(\xi_{u} ; \xi_{\varphi}\right)\right| & \leq 2\left(\sum_{K \in \mathcal{T}} C_{11}^{\partial K}\left\|\xi_{u}\right\|_{0, \partial K}^{2}\right)^{\frac{1}{2}}\left(\sum_{K \in \mathcal{T}} C_{11}^{\partial K}\left\|\xi_{\varphi}\right\|_{0, \partial K}^{2}\right)^{\frac{1}{2}} \\
& \leq S_{5}(\boldsymbol{q}, u ; \boldsymbol{\Phi}, \varphi) .
\end{aligned}
$$

This proves the first assertion. The second one immediately follows by taking into account that

$$
\mathcal{A}\left(\boldsymbol{\xi}_{\boldsymbol{q}}, \xi_{u} ; \boldsymbol{\xi}_{\boldsymbol{q}}, \xi_{u}\right)=a\left(\boldsymbol{\xi}_{\boldsymbol{q}}, \boldsymbol{\xi}_{\boldsymbol{q}}\right)+c\left(\xi_{u}, \xi_{u}\right),
$$

and the proof of the lemma is complete.

The following result is a straightforward consequence of the estimates in Lemma 3.3. Corollary 3.4. Let $(\boldsymbol{q}, u) \in H^{s+1}(\Omega)^{2} \times H^{s+2}(\Omega), s \geq 0$, be the exact solution of (1.2)-(1.5); let $\varphi \in H^{t+2}(\Omega), t \geq 0$, be the solution of the dual problem (2.21)-(2.23), 
and $\mathbf{\Phi}=-\nabla \varphi$. Assume that coefficients $C_{11}$ and $C_{22}$ satisfy (2.15), (2.16) . Then there exist a constant $C$ that solely depends on $\sigma, \zeta, \tau, k$ and $d$ such that

$$
K_{\mathcal{A}}(\boldsymbol{q}, u ; \boldsymbol{\Phi}, \varphi)=C h^{Q_{\mathcal{A}}}\|u\|_{s+2}\|\varphi\|_{t+2},
$$

where $Q_{\mathcal{A}}=1+\alpha$ for $k=0$ and $Q_{\mathcal{A}}=\min \{s+1+\min \{t+\hat{\beta}, k\}, k+1+\min \{t, k+\alpha\}\}$. for $k \geq 1$. Moreover,

$$
K_{\mathcal{A}}(\boldsymbol{q}, u ; \boldsymbol{q}, u)=C h^{2 P_{\mathcal{A}}}\|u\|_{s+2}^{2} .
$$

where $P_{\mathcal{A}}=\frac{1}{2}(1+\alpha)$ for $k=0$ and $P_{\mathcal{A}}=\min \left\{s+\frac{1}{2}(1+\hat{\beta}), k+\frac{1}{2}(1+\alpha)\right\}$ for $k \geq 1$. Proof. From Lemma 3.3, we get

$$
\begin{aligned}
K_{\mathcal{A}}(\boldsymbol{q}, u ; \boldsymbol{\Phi}, \varphi)= & C\left[h^{\min \{s, k\}+1}\left(h^{\min \{t, k\}+1}+\tau h^{\min \{t, k\}+\beta}+h^{\min \{t+1, k\}}\right)\right. \\
& \left.+h^{\min \{s+1, k\}+1}\left(h^{\min \{t, k\}}+\zeta h^{\min \{t+1, k\}+\alpha}\right)\right]\|u\|_{s+2}\|\varphi\|_{t+2},
\end{aligned}
$$

and

$$
K_{\mathcal{A}}(\boldsymbol{q}, u ; \boldsymbol{q}, u)=C\left[h^{2 \min \{s, k\}+1}\left(h+\tau h^{\beta}\right)+h^{2 \min \{s+1, k\}+1} \zeta h^{\alpha}\right]\|u\|_{s+2}^{2} .
$$

Note that the above results hold for arbitrary $\alpha$ and $\beta$. If now we restrict ourselves to the case of Theorem 2.2, the result follows after simple algebraic manipulations. $\square$

3.3. The functional $K_{\mathcal{B}}$. In this subsection we determine the functional $K_{\mathcal{B}}$ satisfying (2.19), up to a multiplicative constant independent of the mesh-size. Here, we take $\Pi$ to be $L^{2}$-projection and $\Pi=(\Pi, \cdots, \Pi)$. Again, we start by determining expressions which are valid for varying coefficients $C_{11}$ and $C_{22}$, and we conclude by considering the particular case of Theorem 2.2. We proceed as follows. We show that there exists a form $|(\cdot, \cdot)|_{\mathcal{B}}$, which is a semi-norm in both variables, such that for any $(\boldsymbol{r}, v) \in \boldsymbol{M}_{N} \times V_{N}$ and $(\boldsymbol{q}, u) \in H^{1}(\Omega)^{d} \times H^{2}(\Omega)$,

$$
|\mathcal{A}(\boldsymbol{r}, v ; \boldsymbol{q}-\Pi \boldsymbol{q}, u-\Pi u)| \leq C|(\boldsymbol{r}, v)|_{\mathcal{A}}|(\boldsymbol{q}-\Pi \boldsymbol{q}, u-\Pi u)|_{\mathcal{B}},
$$

with $C$ independent of the mesh-size. Then it is enough to determine $K_{\mathcal{B}}$ such that

$$
|(\boldsymbol{q}-\Pi \boldsymbol{q}, u-\Pi u)|_{\mathcal{B}} \leq K_{\mathcal{B}}(\boldsymbol{q}, u)
$$

for any $(\boldsymbol{q}, u) \in \boldsymbol{M} \times V$. In the following lemma we prove that (3.1) is satisfied by defining the semi-norm $|(\cdot, \cdot)|_{\mathcal{B}}$ as

$$
\begin{aligned}
& \left.(\boldsymbol{q}, u)\right|_{\mathcal{B}} ^{2}:=\int_{\mathcal{E}_{\mathcal{D}}}\left(\frac{1}{C_{11}}(\boldsymbol{q} \cdot \boldsymbol{n})^{2}+C_{11} u^{2}\right) d s+\int_{\mathcal{E}_{\mathcal{N}}}\left(C_{22}(\boldsymbol{q} \cdot \boldsymbol{n})^{2}+\frac{u^{2}}{\gamma}\right) d s \\
& +\int_{\mathcal{E}_{i}}\left(C_{22} \llbracket \boldsymbol{q} \rrbracket^{2}+\frac{1}{C_{11}} \mid\left\{\boldsymbol{q} \rrbracket-\left.\boldsymbol{C}_{12} \llbracket \boldsymbol{q} \rrbracket\right|^{2}+\frac{1}{\chi}\left(\{u u\}+\boldsymbol{C}_{12} \cdot \llbracket u \rrbracket\right)^{2}+C_{11} \llbracket u \rrbracket^{2}\right) d s,(3 .)\right.
\end{aligned}
$$

where for each internal or Neumann boundary face $e$ we set

$$
\chi(\boldsymbol{x}):= \begin{cases}\min \left\{h_{K}, h_{K^{\prime}}\right\} \text { for } \boldsymbol{x} \in\left\langle K, K^{\prime}\right\rangle, h_{K} \text { for } \boldsymbol{x} \in \Gamma_{\mathcal{N}} & \text { if } C_{22}(\boldsymbol{x})=0, \\ C_{22}(\boldsymbol{x}) & \text { otherwise. }\end{cases}
$$

Note that only the function values along faces enter the $|(\cdot, \cdot)|_{\mathcal{B}}$ semi-norm. As can be inferred from the proof of Lemma 3.5 below, this is due to the particular choice of $\Pi$ and $\Pi$ as $L^{2}$-projections. 
Lemma 3.5. Let $\Pi$ and $\Pi$ be the $L^{2}(\Omega)$-projection and $L^{2}(\Omega)^{d}$-projection onto $V_{N}$ and $\boldsymbol{M}_{N}$, respectively, and $|(\cdot, \cdot)|_{\mathcal{B}}$ be defined by (3.3). Then (3.1) holds true, with a constant $C$ that solely depends on $\sigma, k$ and $d$.

Proof. Setting $\boldsymbol{\xi}_{\boldsymbol{q}}:=\boldsymbol{q}-\boldsymbol{\Pi} \boldsymbol{q}$ and $\xi_{u}=u-\Pi u$, we have, by the definition of the form $\mathcal{A}$ in (2.9),

$$
\begin{aligned}
\left|\mathcal{A}\left(\boldsymbol{r}, v ; \boldsymbol{\xi}_{\boldsymbol{q}}, \xi_{u}\right)\right| & \leq\left|a\left(\boldsymbol{r}, \boldsymbol{\xi}_{\boldsymbol{q}}\right)\right|+\left|b\left(v, \boldsymbol{\xi}_{\boldsymbol{q}}\right)\right|+\left|b\left(\xi_{u}, \boldsymbol{r}\right)\right|+\left|c\left(v, \xi_{u}\right)\right| \\
& =: T_{1}+T_{2}+T_{3}+T_{4} .
\end{aligned}
$$

Using Cauchy-Schwarz's inequality and the fact that $\Pi$ is the $L^{2}(\Omega)^{d}$-projection, we obtain

$$
\begin{aligned}
T_{1} & \leq\left(\int_{\mathcal{E}_{i}} C_{22} \llbracket \boldsymbol{r} \rrbracket^{2} d s+\int_{\mathcal{E}_{\mathcal{N}}} C_{22}(\boldsymbol{r} \cdot \boldsymbol{n})^{2} d s\right)^{\frac{1}{2}} \cdot\left(\int_{\mathcal{E}_{i}} C_{22} \llbracket \boldsymbol{\xi}_{\boldsymbol{q}} \rrbracket^{2} d s+\int_{\mathcal{E}_{\mathcal{N}}} C_{22}\left(\boldsymbol{\xi}_{\boldsymbol{q}} \cdot \boldsymbol{n}\right)^{2} d s\right)^{\frac{1}{2}} \\
& \leq|(\boldsymbol{r}, v)|_{\mathcal{A}}\left|\left(\boldsymbol{\xi}_{\boldsymbol{q}}, \xi_{u}\right)\right|_{\mathcal{B}} .
\end{aligned}
$$

Furthermore,

$$
T_{2}=\mid \int_{\mathcal{E}_{i}} \llbracket v \rrbracket \cdot\left(\left\{\boldsymbol{\xi}_{\boldsymbol{q}} \rrbracket-\boldsymbol{C}_{12} \llbracket \boldsymbol{\xi}_{\boldsymbol{q}} \rrbracket\right) d s+\int_{\mathcal{E}_{\mathcal{D}}} v \boldsymbol{\xi}_{\boldsymbol{q}} \cdot \boldsymbol{n} d s \mid .\right.
$$

Multiplying and dividing by $C_{11}^{\frac{1}{2}}$ and then applying Cauchy-Schwarz's inequality, we obtain

$$
\begin{aligned}
T_{2} \leq & \left(\int_{\mathcal{E}_{i}} C_{11} \llbracket v \rrbracket^{2} d s+\int_{\mathcal{E}_{\mathcal{D}}} C_{11} v^{2} d s\right)^{\frac{1}{2}} \\
& \cdot\left(\int_{\mathcal{E}_{i}} \frac{1}{C_{11}}\left|\left\{\boldsymbol{\xi}_{\boldsymbol{q}}\right\}-\boldsymbol{C}_{12} \llbracket \boldsymbol{\xi}_{\boldsymbol{q}} \rrbracket\right|^{2} d s+\int_{\mathcal{E}_{\mathcal{D}}} \frac{1}{C_{11}}\left(\boldsymbol{\xi}_{\boldsymbol{q}} \cdot \boldsymbol{n}\right)^{2} d s\right)^{\frac{1}{2}} \\
\leq & |(\boldsymbol{r}, v)|_{\mathcal{A}}\left|\left(\boldsymbol{\xi}_{\boldsymbol{q}}, \xi_{u}\right)\right|_{\mathcal{B} .} .
\end{aligned}
$$

Analogously,

$$
\begin{aligned}
T_{3}= & \left|\int_{\mathcal{E}_{i}}\left(\left\{\xi_{u} \rrbracket\right\}+\boldsymbol{C}_{12} \cdot \llbracket \xi_{u} \rrbracket\right) \llbracket r \rrbracket d s+\int_{\mathcal{E}_{\mathcal{N}}} \xi_{u} \boldsymbol{r} \cdot \boldsymbol{n} d s\right| \\
\leq & \left(\int_{\mathcal{E}_{i}} \chi \llbracket r \rrbracket^{2} d s+\int_{\mathcal{E}_{\mathcal{N}}} \chi(\boldsymbol{r} \cdot \boldsymbol{n})^{2} d s\right)^{\frac{1}{2}} \\
& \cdot\left(\int _ { \mathcal { E } _ { i } } \frac { 1 } { \chi } \left(\left\{\left\{\xi_{u} \rrbracket+\boldsymbol{C}_{12} \cdot \llbracket \xi_{u} \rrbracket\right)^{2} d s+\int_{\mathcal{E}_{\mathcal{N}}} \frac{\xi_{u}{ }^{2}}{\chi} d s\right)^{\frac{1}{2}} .\right.\right.
\end{aligned}
$$

The first factor can be estimated as follows:

$$
\begin{aligned}
\int_{\mathcal{E}_{i}} \chi \llbracket r \rrbracket^{2} d s+\int_{\mathcal{E}_{\mathcal{N}}} \chi(\boldsymbol{r} \cdot \boldsymbol{n})^{2} d s & \leq \int_{\mathcal{E}_{i}} C_{22} \llbracket \boldsymbol{r} \rrbracket^{2} d s+\int_{\mathcal{E}_{\mathcal{N}}} C_{22}(\boldsymbol{r} \cdot \boldsymbol{n})^{2} d s \\
& +\int_{\mathcal{E}_{i}} \bar{\chi} \llbracket \boldsymbol{r} \rrbracket^{2} d s+\int_{\mathcal{E}_{\mathcal{N}}} \bar{\chi}(\boldsymbol{r} \cdot \boldsymbol{n})^{2} d s \\
& \leq|(\boldsymbol{r}, v)|_{\mathcal{A}}^{2}+\int_{\mathcal{E}_{i}} \bar{\chi} \llbracket \boldsymbol{r} \rrbracket^{2} d s+\int_{\mathcal{E}_{\mathcal{N}}} \bar{\chi}(\boldsymbol{r} \cdot \boldsymbol{n})^{2} d s
\end{aligned}
$$


where $\bar{\chi}(\boldsymbol{x})=\min \left\{h_{K}, h_{K^{\prime}}\right\}$ if $\boldsymbol{x} \in\left\langle K, K^{\prime}\right\rangle$, and $\bar{\chi}(\boldsymbol{x})=h_{K}$ if $\boldsymbol{x} \in \Gamma_{\mathcal{N}}$. By the inverse inequality in Lemma 3.2,

$$
\begin{aligned}
\int_{\mathcal{E}_{i}} \bar{\chi} \llbracket \boldsymbol{r} \rrbracket^{2} d s+\int_{\mathcal{E}_{\mathcal{N}}} \bar{\chi}(\boldsymbol{r} \cdot \boldsymbol{n})^{2} d s & \leq\left.\sum_{K \in \mathcal{T}} \sum_{e \in \partial K} \int_{e} 2 \bar{\chi}|\boldsymbol{r}|_{K} \cdot \boldsymbol{n}\right|^{2} d s \\
& \leq \sum_{K \in \mathcal{T}} 2 \bar{\chi}^{\partial K}\left\|\left.\boldsymbol{r}\right|_{K} \cdot \boldsymbol{n}\right\|_{0, \partial K}^{2} \\
& \leq 2 C_{\mathrm{inv}} \sup _{K \in \mathcal{T}} \frac{\bar{\chi}^{\partial K}}{h_{K}}\|\boldsymbol{r}\|_{0}^{2} \\
& \leq 2 C_{\mathrm{inv}}\|\boldsymbol{r}\|_{0}^{2} \leq 2 C_{\mathrm{inv}}|(\boldsymbol{r}, v)|_{\mathcal{A}}^{2},
\end{aligned}
$$

where $\bar{\chi}^{\partial K}=\sup \{\bar{\chi}(\boldsymbol{x}): \boldsymbol{x} \in \partial K\}$. Thus, combining the above estimates, we get

$$
T_{3} \leq C|(\boldsymbol{r}, v)|_{\mathcal{A}}\left|\left(\boldsymbol{\xi}_{\boldsymbol{q}}, \xi_{u}\right)\right|_{\mathcal{B}}
$$

Finally,

$$
\begin{aligned}
T_{4} & =\left|\int_{\mathcal{E}_{i}} C_{11} \llbracket v \rrbracket \cdot \llbracket \xi_{u} \rrbracket d s+\int_{\mathcal{E}_{\mathcal{D}}} C_{11} v \xi_{u} d s\right| \\
& \leq\left(\int_{\mathcal{E}_{i}} C_{11} \llbracket v \rrbracket^{2} d s+\int_{\mathcal{E}_{\mathcal{D}}} C_{11} v^{2} d s\right)^{\frac{1}{2}} \cdot\left(\int_{\mathcal{E}_{i}} C_{11} \llbracket \xi_{u} \rrbracket^{2} d s+\int_{\mathcal{E}_{\mathcal{D}}} C_{11} \xi_{u}{ }^{2} d s\right)^{\frac{1}{2}} \\
& \leq|(\boldsymbol{r}, v)|_{\mathcal{A}}\left|\left(\boldsymbol{\xi}_{\boldsymbol{q}}, \xi_{u}\right)\right|_{\mathcal{B} .}
\end{aligned}
$$

To complete the proof, we simply have to gather the estimates of the terms $T_{i}, i=$ $1,2,3,4$, and apply once again the Cauchy-Schwarz's inequality.

The function $K_{\mathcal{B}}$ can be easily defined by applying the estimates in Lemma 3.1 to $|(\cdot, \cdot)|_{\mathcal{B}}$ defined in $(3.3)$.

Lemma 3.6. For any $(\boldsymbol{q}, u) \in H^{s+1}(\Omega)^{2} \times H^{s+2}(\Omega), s \geq 0$, the approximation property (3.2) holds true with

$$
\begin{aligned}
K_{\mathcal{B}}^{2}(\boldsymbol{q}, u)= & C \sum_{K \in \mathcal{T}}\left(h_{K}^{2 \min \{s, k\}+1}\left(\frac{1}{\widetilde{C}_{11}^{\partial K}}+C_{22}^{\partial K}\right)\|\boldsymbol{q}\|_{s+1, K}^{2}\right) \\
& +C \sum_{K \in \mathcal{T}}\left(h_{K}^{2 \min \{s+1, k\}+1}\left(C_{11}^{\partial K}+\frac{1}{\widetilde{\chi}^{\partial K}}\right)\|u\|_{s+2, K}^{2}\right),
\end{aligned}
$$

where $\widetilde{C}_{11}^{\partial K}=\inf \left\{C_{11}(\boldsymbol{x}): \boldsymbol{x} \in \partial K\right\}, \widetilde{\chi}^{\partial K}=\inf \{\chi(\boldsymbol{x}): \boldsymbol{x} \in \partial K\}$, and $C$ is a constant independent of the mesh-size and solely depending on the approximation and inverse inequality constants (cf. Lemmas 3.1 and 3.2).

From this lemma, we immediately obtain the following result.

Corollary 3.7. Let $(\boldsymbol{q}, u) \in H^{s+1}(\Omega)^{2} \times H^{s+2}(\Omega), s \geq 0$. Assume that the coefficients $C_{11}$ and $C_{22}$ satisfy (2.15), (2.16). The triangulations are assumed to satisfy the hypothesis (2.11); if $\alpha \neq 0$ or $\beta \neq 0$, we also assume that hypothesis (2.12) is satisfied.

Then there exists a constant $C$ that solely depends on $\sigma, \delta, \zeta, \tau, k$ and $d$ such that

$$
K_{\mathcal{B}}^{2}(\boldsymbol{q}, u)=C h^{2 P}\|u\|_{s+2}^{2},
$$

where $P=\frac{1}{2}\left(1-\mu^{\star}\right)$ if $k=0$ and $P=\min \left\{s+\frac{1}{2}\left(1+\mu_{\star}\right), k+\frac{1}{2}\left(1-\mu^{\star}\right)\right\}$, if $k \geq 1$. If $\alpha=\beta=0$ the constant $C$ is independent of $\delta$. 
Proof. If we take the coefficients $C_{11}$ and $C_{22}$ as in Theorem 2.2, we get, after a simple computation,

$$
\left(\frac{1}{\widetilde{C}_{11}^{\partial K}}+C_{22}^{\partial K}\right) \leq \zeta^{-1} h_{K}^{-\alpha} \delta^{\alpha}+\tau h_{K}^{\beta},
$$

and

$$
\left(C_{11}^{\partial K}+\frac{1}{\widetilde{\chi}^{\partial K}}\right) \leq \zeta h_{K}^{\alpha}+\widehat{\tau}^{-1} h_{K}^{-\widehat{\beta}} \delta^{-\widehat{\beta}}
$$

where the parameter $\delta$ is defined in (2.12), and $\widehat{\tau}=1$ if $\tau=0, \widehat{\tau}=\tau$ otherwise. Note that the left-hand sides of the above inequalities are trivially uniformly bounded when $\alpha=\beta=0$; otherwise, we must invoke the hypothesis (2.12) to ensure the boundedness of these quantities. We emphasize that this is the only instance in which this hypothesis is used.

Hence we obtain

$$
K_{\mathcal{B}}^{2}(\boldsymbol{q}, u)=C\left[h^{2 \min \{s, k\}+1}\left(\zeta^{-1} h^{-\alpha}+\tau h^{\beta}\right)+h^{2 \min \{s+1, k\}+1}\left(\zeta h^{\alpha}+\overline{\bar{\chi}}^{-1}\right)\right]\|u\|_{s+2}^{2},
$$

where $C$ is independent of the mesh-size but depends on $\delta$ and on the approximation and inverse inequality constants, and

$$
\overline{\bar{\chi}}:= \begin{cases}h & \text { if } \tau=0, \\ \tau h^{\beta} & \text { otherwise. }\end{cases}
$$

The result follows after simple algebraic manipulations.

3.4. The proof of Theorem 2.2. From Lemma 2.3 and Corollaries 3.4 and 3.7 , we get

$$
\left|\left(\boldsymbol{q}-\boldsymbol{q}_{N}, u-u_{N}\right)\right|_{\mathcal{A}} \leq C h^{\min \left\{P_{\mathcal{A}}, P\right\}}\|u\|_{s+2},
$$

and since $\min \left\{P_{\mathcal{A}}, P\right\}=P$ the estimate

$$
\left|\left(\boldsymbol{q}-\boldsymbol{q}_{N}, u-u_{N}\right)\right|_{\mathcal{A}} \leq C h^{P}\|u\|_{s+2}
$$

follows.

Next, consider the $L^{2}$-norm of the error $u-u_{N}$. Take $t=0$ and $D=\Omega$ in Lemma 2.4. From the elliptic regularity of the adjoint problem (2.21)-(2.23), we have $\|\varphi\|_{2} \leq C\|\lambda\|_{0}$ and $\|\boldsymbol{\Phi}\|_{1} \leq C\|\lambda\|_{0}$. The estimates of $\left\|u-u_{N}\right\|_{0}$ directly follow from substituting the expression of $K_{\mathcal{A}}(\boldsymbol{q}, u ; \boldsymbol{\Phi}, \varphi)$ given by Corollary 3.4, and the expressions of $K_{\mathcal{B}}(\boldsymbol{q}, u), K_{\mathcal{B}}(\boldsymbol{\Phi}, \varphi)$ given by Corollary 3.7 in (2.24), and bounding $\|\boldsymbol{\Phi}\|_{1}$ and $\|\varphi\|_{2}$ by $\|\lambda\|_{0}$. Indeed, we get

$$
\left\|u-u_{N}\right\|_{0} \leq C h^{\min \left\{\left.Q_{\mathcal{A}}\right|_{t=0}, P+\left.P\right|_{s=0}\right\}}\|u\|_{s+2},
$$

and since $\min \left\{\left.Q_{\mathcal{A}}\right|_{t=0}, P+\left.P\right|_{s=0}\right\}=P+\left.P\right|_{s=0}$, the estimate $\left\|u-u_{N}\right\|_{0} \leq C h^{P+D}$ follows with $D=\left.P\right|_{s=0}$.

This completes the proof of Theorem 2.2.

4. Extensions. In this section, we indicate how to the extend our main result in several possible directions. 
4.1. The case of polygonal domains. In the case of a non-convex polygonal domain in two dimensions, our assumptions on the smoothness of the solution $u$ of our model problem (1.1) and on the elliptic regularity inequality are no longer true. Indeed, if for instance the Neumann boundary is empty, the Dirichlet data is smooth and $f$ is in $\mathrm{L}^{2}(\Omega)$, we have, see Grisvard [28], that $u \in H^{s+2}(\Omega)$ with $s=\gamma-1 \in$ $(-1 / 2,0)$, where

$$
\gamma=\pi / \omega
$$

and $\omega$ is the maximum interior angle of $\partial \Omega$. Moreover, if the Dirichlet data is zero, we have

$$
\|u\|_{s+2-\varepsilon} \leq C(\varepsilon)\|f\|_{0}, \forall \varepsilon>0 ;
$$

see (1.7) in Schatz and Wahlbin [37] and the references therein. This is the elliptic regularity result that we must use.

To prove our error estimates in this case, we proceed as follows. First, we note that our main result Theorem 2.2 can be easily extended to this case; indeed, a simple density argument shows that Lemmas 3.3 and 3.6 remain valid for $s, t \in(-1 / 2,0)$. Now we proceed as in subsection 3.4 and obtain the desired estimates by using the above mentioned lemmas and the above described elliptic regularity inequality. The estimate of the error in the $|(\cdot, \cdot)|_{\mathcal{A}^{-}}$-seminorm remains the same but the estimate of the $\mathrm{L}^{2}$-norm of the potential has to be suitably modified.

For $k=0$, it turns out that only for $\alpha=\beta=0$ we obtain non-zero orders of convergence for $\left|\left(\boldsymbol{q}-\boldsymbol{q}_{N}, u-u_{N}\right)\right|_{\mathcal{A}}$ and $\left\|u-u_{N}\right\|_{0}$, namely, $\gamma-1 / 2-\varepsilon$ and $2 \gamma-1-\varepsilon$ for all $\varepsilon>0$, respectively. The results for $k \geq 1$ are displayed in Table 4.1 for smooth solutions $\left(u \in H^{s+2}(\Omega), s \geq 0\right)$ and in Table 4.2 for non-smooth solutions $\left(u \in H^{s+2}(\Omega), s=\gamma-1\right)$. (We simply write $\gamma$ instead of $\gamma-\varepsilon, \forall \varepsilon>0$.)

TABLE 4.1 Orders of convergence for $u \in H^{s+2}(\Omega)$ for $s \geq 0$ and $k \geq 1$.

\begin{tabular}{cc|c|c}
\hline$C_{22}$ & $C_{11}$ & $\left|\left(\boldsymbol{q}-\boldsymbol{q}_{N}, u-u_{N}\right)\right|_{\mathcal{A}}$ & $\left\|u-u_{N}\right\|_{0}$ \\
\hline $\begin{array}{cc}0, \mathcal{O}(h) \\
0, \mathcal{O}(h)\end{array}$ & $\mathcal{O}(1)$ & $\begin{array}{c}\min \{s+1 / 2, k\} \\
\min \{s+1, k\}\end{array}$ & $\begin{array}{c}\min \{s+1 / 2, k\}+\gamma-1 / 2 \\
\min \{s+1, k\}+\gamma\end{array}$ \\
\hline $\mathcal{O}(1)$ & $\mathcal{O}(1)$ & $\min \{s, k\}+1 / 2$ & $\min \{s, k\}+\gamma-1 / 2$ \\
$\mathcal{O}(1)$ & $\mathcal{O}(1 / h)$ & $\min \{s+1 / 2, k\}$ & $\min \{s+1 / 2, k\}+\gamma-1 / 2$ \\
\hline
\end{tabular}

4.2. Estimates of the error in negative-order norms. It is very well known that the error in linear functionals can be estimated in terms of the error in negativeorder norms. Moreover, Bramble and Schatz [11] showed how to exploit the oscillatory nature of finite element approximations, captured in estimates of the error in negativeorder norms, to enhance the quality of the approximation by using a simple postprocessing on regions in which the exact solution is very smooth and the mesh is locally translation invariant. 
TABLE 4.2

Orders of convergence for $u \in H^{s+2}(\Omega)$ for $s=\gamma-1 \in(-1 / 2,0)$ and $k \geq 1$.

\begin{tabular}{cc|c|c}
\hline$C_{22}$ & $C_{11}$ & $\left|\left(\boldsymbol{q}-\boldsymbol{q}_{N}, u-u_{N}\right)\right|_{\mathcal{A}}$ & $\left\|u-u_{N}\right\|_{0}$ \\
\hline \multirow{2}{*}{$\mathcal{O}(h)$} & $\mathcal{O}(1)$ & $\gamma-1 / 2$ & $2 \gamma-1$ \\
$0, \mathcal{O}(h)$ & $\mathcal{O}(1 / h)$ & $\gamma$ & $2 \gamma$ \\
& & & \\
\hline $\mathcal{O}(1)$ & $\mathcal{O}(1)$ & $\gamma-1 / 2$ & $2 \gamma-1$ \\
$\mathcal{O}(1)$ & $\mathcal{O}(1 / h)$ & $\gamma-1 / 2$ & $2 \gamma-1$ \\
\hline
\end{tabular}

Error estimates of negative-order norms can be easily obtained for our general DG method by following the argument described in subsection 2.4 and the technicalities displayed in section 3 .

4.3. Curvilinear elements. The analysis in section 3 covers the case of triangulations of curvilinear elements affine-equivalent to fixed curvilinear reference elements. The aim of this subsection is to show how our main result can be extended to the more general case where such an affine equivalence can not be established anymore. This is, for instance, the case when the problem domain has a boundary with a generic curvature.

There are two distinctive possibilities to do that. The first one is to keep the finite element spaces described in the introduction; in this case, the local space $\mathcal{S}(K)$ could be taken to be simply $\mathcal{P}^{k}(K)$, for example. For our main result to hold in this case, only Lemmas 3.1 and 3.2 would have to be proven for these elements and for the case in which $\Pi$ is the $\mathrm{L}^{2}$-projection.

The other possibility is to consider elements obtained through the so-called Piola transformation [12, Section III.1.3]. This transformation associates the function $(\boldsymbol{q}, u)$ defined on $K$ to the function $(\widehat{\boldsymbol{q}}, \widehat{u})$ defined on $\widehat{K}$ by

$$
\boldsymbol{q}=\left(\frac{1}{\left|\operatorname{det} D F_{K}\right|} D F_{K} \widehat{\boldsymbol{q}}\right) \circ F_{K}^{-1}, \quad u=\widehat{u} \circ F_{K}^{-1}
$$

where $F_{K}$ denotes the mapping from $\widehat{K}$ to $K$. With the above notation, our finite element spaces are given by

$$
\begin{aligned}
& \boldsymbol{M}_{N}:=\left\{\boldsymbol{q} \in\left(L^{2}(\Omega)\right)^{d}: \widehat{\left.\boldsymbol{q}\right|_{K}} \in \mathcal{S}^{d}(\widehat{K}) \quad \forall K \in \mathcal{T}\right\}, \\
& V_{N}:=\left\{u \in L^{2}(\Omega): \widehat{\left.u\right|_{K}} \in \mathcal{S}(\widehat{K}) \quad \forall K \in \mathcal{T}\right\} .
\end{aligned}
$$

It is easy to verify that the following properties are satisfied on each element $K$ of 
our triangulation:

$$
\begin{aligned}
& \int_{K} \boldsymbol{q} \nabla v d x=\int_{\widehat{K}} \widehat{\boldsymbol{q}} \widehat{\nabla} \widehat{v} d \widehat{x}, \\
& \int_{K} \nabla \cdot \boldsymbol{q} v d x=\int_{\widehat{K}} \widehat{\nabla} \cdot \widehat{\boldsymbol{q}} \widehat{v} d \widehat{x}, \\
& \int_{\partial K} \boldsymbol{q} \cdot \boldsymbol{n} v d x=\int_{\partial \widehat{K}} \widehat{\boldsymbol{q}} \cdot \widehat{\boldsymbol{n}} \widehat{v} d \widehat{x} .
\end{aligned}
$$

This implies that with this choice of finite element spaces, our main result holds if Lemmas 3.1 and 3.2 hold for the reference element $\widehat{K}$ and for the standard $\mathrm{L}^{2}$-projection, provided the mappings $F_{K}$ are sufficiently smooth; see [12] and the references therein. Indeed, the proof of section 3 holds in this case if we use the projections $\Pi$ and $\Pi$ defined by

$$
\widehat{\Pi} \boldsymbol{q}:=\widehat{\Pi} \widehat{\boldsymbol{q}}, \quad \widehat{\Pi u}=\widehat{\Pi} \widehat{u},
$$

where $\widehat{\Pi}$ is the $L^{2}$-projection into the space $\mathcal{S}(\widehat{K})$ and $\widehat{\Pi}=(\widehat{\Pi}, \cdots, \widehat{\Pi})$. The only slight modification of the proof occurs in section 3.3 in the definition of $|(\cdot, \cdot)|_{\mathcal{B}}^{2},(3.3)$, to which we have to $a d d$ the term $\|\boldsymbol{q}\|_{0}^{2}$. This implies that an extra term in the upper bound of the term $T_{1}$ in the proof of Lemma 3.5 appears which is easily dealt with. No other modification of the proof is required at all.

4.4. General elliptic problems. The extension of our main result to more general elliptic problems which include lower order terms can be done in a straightforward way by applying our techniques to the formulation used by Cockburn and Dawson [18].

4.5. Exponential convergence of $h p$-approximations. In the analysis of the DG methods considered in this paper, we have only derived error estimates with respect to the mesh-size $h$ and we have not exploited the dependence of our estimates on the approximation order $k$. However, this can be done by modifying Lemmas 3.3 and 3.6 correspondingly; see also the work of Houston, Schwab and Süli [38] and the references there. In addition, by using the proper mesh design principles and by obtaining suitable approximation error estimates in the elements abutting at solution singularities, exponential convergence of the DG method can be proved. See, for example, the recent work of Wihler and Schwab [40] who showed exponential convergence for a model elliptic problem on a polygonal domain $\Omega$ for the DG method of Baumann and Oden with interior penalties.

5. Numerical results for the LDG method. The purpose of this section is to validate our a priori error estimates for the LDG method (i.e., $C_{22}=0$ ) and to assess how the quality of its approximations depends on the size of the stabilization parameters $C_{11}$. Since $C_{22}=0$, the function $\boldsymbol{q}_{N}$ can be expressed locally in terms of $u_{N}$ and hence can be eliminated from the equations. In our examples we solve the resulting linear system for $u_{N}$ by using the standard Conjugate Gradient algorithm; in order to obtain as much precision as possible, the stopping criterion is such that the absolute residual norm is less than $10^{-12}$. The approximation $\boldsymbol{q}_{N}$ is then recovered in a post-processing step by using the local expression of $\boldsymbol{q}_{N}$ in terms of $u_{N}$.

We present numerical results using sequences of structured as well as unstructured triangular meshes $\left\{\mathcal{T}_{i}\right\}, i=1,2, \ldots$, where the mesh-size parameter of $\mathcal{T}_{i+1}$ is half the one of $\mathcal{T}_{i}$. The numerical orders of convergence of the errors are computed for 
polynomials of degree 1 to 6 in the $\mathrm{L}^{2}$-norm and $\mathcal{A}$-semi-norm. These orders are defined as follows. If $e\left(\mathcal{T}_{i}\right)$ denotes the error on mesh $\mathcal{T}_{i}$ (in the corresponding norm), then the numerical order of convergence $r_{i}$ is

$$
r_{i}=\log \left(\frac{e\left(\mathcal{T}_{i+1}\right)}{e\left(\mathcal{T}_{i}\right)}\right) / \log (0.5) .
$$

In all our computations, we take $\boldsymbol{C}_{12}$ normal to the edges and of modulus $1 / 2$. The stabilization coefficient $C_{11}$ is chosen to be of order $h^{-1}$. We emphasize, however, that for all our experiments no significant difference has been observed in the errors of the approximations when $C_{11}$ is of order one. We also remark that results for $k=0$ are not included either, since no positive orders of convergence have been obtained, as predicted in Theorem 2.2.

5.1. Smooth solutions. In our first example, we investigate the order of convergence for smooth solutions. We solve the model problem $(1.1)$ in $\Omega=(-1,1) \times(-1,1)$ with homogeneous Dirichlet boundary conditions and empty Neumann boundary. The right hand side $f$ is chosen such that the exact solution is given by

$$
u(x, y)=\cos \left(\frac{\pi}{2} x\right) \cos \left(\frac{\pi}{2} y\right) .
$$

The sequence of structured meshes used in this example is created from consecutive global refinement of an initial coarse structured mesh; at each refinement, every triangle is divided into 4 similar triangles. The number of triangles of the meshes are $16,64,256,1024$ and 4096. Since our analysis is valid for arbitrary meshes, we also perform some tests with a sequence of unstructured meshes. It consists of a set of meshes such that the maximum edge length is less than a certain value. This value is reduced by a factor of two, from one mesh to the next. In this way, if we take two consecutive meshes, one is not the global refinement of the other. The number of elements of the meshes are 22, 88, 312, 1368 and 5404 .

We show the orders of convergence in the $\mathrm{L}^{2}$-norm of the error in the gradient $\boldsymbol{q}=\nabla u$, in the $\mathcal{A}$-semi-norm of the error of $(\boldsymbol{q}, u)$ and in the $\mathrm{L}^{2}$-norm of the error in $u$ in Tables 5.1, 5.2 and 5.3, respectively. For both types of meshes, we observe that the optimal order of convergence predicted by our theory, see Table 2.2 , is achieved. Note that since machine precision is achieved for very fine grids and high polynomials, the corresponding orders of convergence are meaningless and are replaced by a horizontal line.

To give the reader a better idea of this phenomenon, in Figure 5.1, we display the actual $\mathrm{L}^{2}$ errors in the potential $u$ whose orders of convergence appear in the left side of Table 5.3. Note how the very last part of the curve corresponding to polynomials of degree $k=6$ bends as a consequence of having reached machine accuracy. 
TABle 5.1

Smooth solution; order of convergence of the $L^{2}$ error in the gradient $\boldsymbol{q}$.

\begin{tabular}{c|c|c|c|c||c|c|c|c}
\hline$k$ & \multicolumn{8}{|c}{ order of convergence } \\
\hline & \multicolumn{3}{|c}{ Structured meshes } & \multicolumn{4}{c}{ Unstructured meshes } \\
\hline 1 & 0.7075 & 0.9424 & 0.9809 & 0.9932 & 0.9585 & 0.7681 & 1.1016 & 0.9505 \\
2 & 1.9701 & 1.9764 & 1.9864 & 1.9925 & 2.0364 & 1.6525 & 2.0644 & 1.9843 \\
3 & 2.7216 & 2.9488 & 2.9924 & 3.0008 & 2.9303 & 2.4986 & 3.2825 & 2.9120 \\
4 & 3.9171 & 3.9723 & 3.9853 & 3.9920 & 4.2473 & 3.3587 & 4.2419 & 3.9563 \\
5 & 4.7384 & 4.9438 & 4.9897 & 5.0114 & 4.8836 & 4.2077 & 5.5068 & 4.8490 \\
6 & 5.8878 & 5.9683 & 5.9820 & - & 6.4090 & 5.0744 & 6.4362 & - \\
\hline
\end{tabular}

TABle 5.2

Smooth solution; order of convergence of the $\mathcal{A}$-semi-norm of the error in $(\boldsymbol{q}, u)$.

\begin{tabular}{c|c|c|c|c||c|c|c|c}
\hline$k$ & \multicolumn{7}{|c}{ order of convergence } \\
\hline & \multicolumn{3}{|c}{ Structured meshes } & \multicolumn{4}{c}{ Unstructured meshes } \\
\hline 1 & 0.8703 & 0.9762 & 0.9942 & 0.9996 & 1.0346 & 0.8469 & 1.1349 & 0.9692 \\
2 & 2.0661 & 2.0068 & 1.9995 & 1.9988 & 2.1175 & 1.7205 & 2.0926 & 1.9949 \\
3 & 2.8380 & 2.9745 & 3.0018 & 3.0052 & 3.0120 & 2.5618 & 3.2984 & 2.9233 \\
4 & 4.0002 & 3.9938 & 3.9943 & 3.9963 & 4.3158 & 3.4195 & 4.2588 & 3.9632 \\
5 & 4.8276 & 4.9634 & 4.9965 & 5.0144 & 4.9972 & 4.2627 & 5.5138 & 4.8575 \\
6 & 5.9582 & 5.9844 & 5.9885 & - & 6.4724 & 5.1322 & 6.4472 & - \\
\hline
\end{tabular}

TABle 5.3

Smooth solution; order of convergence of the $L^{2}$ error in the potential $u$.

\begin{tabular}{c|c|c|c|c||c|c|c|c}
\hline$k$ & \multicolumn{6}{|c}{ order of convergence } \\
\hline & \multicolumn{4}{|c}{ Structured meshes } & \multicolumn{4}{c}{ Unstructured meshes } \\
\hline 1 & 1.7916 & 1.9471 & 1.9855 & 1.9956 & 1.8655 & 1.7322 & 2.1761 & 1.9562 \\
2 & 2.9241 & 2.9872 & 2.9868 & 2.9915 & 2.9656 & 2.7066 & 3.1625 & 2.9828 \\
3 & 4.0140 & 3.9837 & 3.9891 & 3.9942 & 3.8730 & 3.5958 & 4.2955 & 3.9421 \\
4 & 4.9796 & 4.9830 & 4.9866 & 4.9922 & 5.1529 & 4.5272 & 5.3216 & 4.9585 \\
5 & 6.0358 & 5.9949 & 5.9910 & 5.9589 & 5.9806 & 5.4050 & 6.4370 & 5.7302 \\
6 & 7.0129 & 6.9889 & 6.8763 & - & 7.3003 & 6.3641 & 6.8498 & - \\
\hline
\end{tabular}




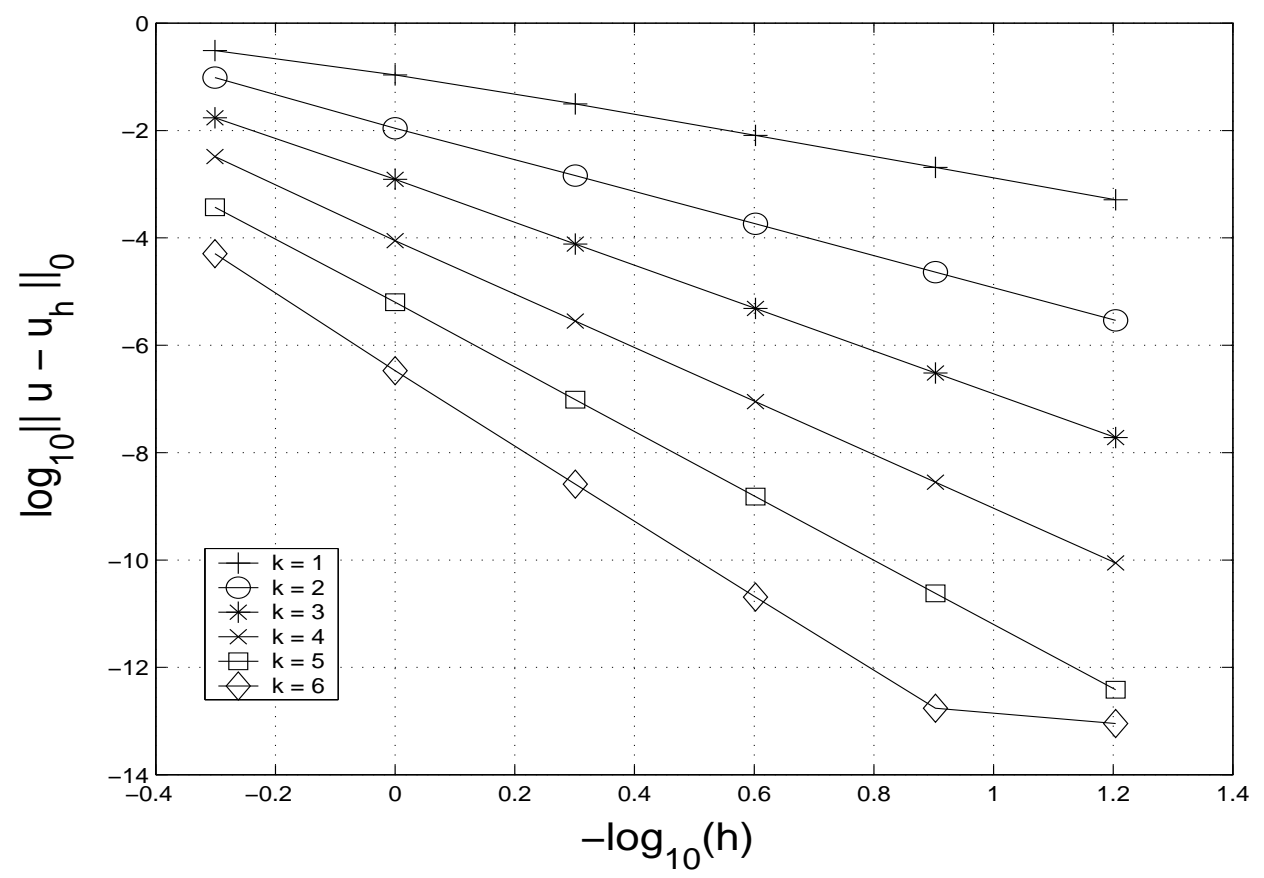

FIG. 5.1. Smooth solution; the $L^{2}$ error in the potential $u$ for the structured meshes. 
5.2. An exact solution in $H^{5}(\Omega)$ but not in $H^{5+\varepsilon}(\Omega) \forall \varepsilon>0$. We solve the model problem (1.1) with exact Dirichlet boundary conditions in the convex domain $\Omega=(-1,1) \times(-1,1)$. The right hand side is chosen such that the exact solution of the problem is the function $u_{\alpha}$ defined by

$$
u_{\alpha}(x, y)= \begin{cases}\cos \left(\frac{\pi}{2} y\right) & \text { in }(-1,0) \times(-1,1) \\ \cos \left(\frac{\pi}{2} y\right)+x^{\alpha} & \text { in }(0,1) \times(-1,1)\end{cases}
$$

This function belongs to $H^{\alpha+\frac{1}{2}}(\Omega)$ but does not belong to $H^{\alpha+\frac{1}{2}+\varepsilon}(\Omega)$, for all $\varepsilon>0$. In this test, $\alpha=4.5$ and so $u_{\alpha} \in H^{5}(\Omega)$. The predicted orders of convergence of the $\mathrm{L}^{2}$-norm of the error in the gradient and that of the $\mathcal{A}$-semi-norm of the error are both 5 , and the predicted order of convergence of the $\mathrm{L}^{2}$-norm of the error in the potential is 4; see Tables 2.2 and 2.3. These are precisely the orders observed in Tables 5.4, 5.5 and 5.6, respectively. We use the sequence of structured meshes from the previous test. Similar results not reported here are obtained using unstructured meshes.

TABLE 5.4

$H^{5}$-solution; order of convergence of the $L^{2}$ error in the gradient $\boldsymbol{q}$.

\begin{tabular}{c|c|c|c|c}
\hline$k$ & \multicolumn{4}{|c}{ order of convergence } \\
\hline 1 & 0.9198 & 0.8929 & 0.9598 & 0.9872 \\
2 & 1.3322 & 1.7872 & 1.9288 & 1.9756 \\
3 & 2.1363 & 2.8375 & 2.9531 & 2.9844 \\
4 & 3.7141 & 3.8566 & 3.9005 & 3.9213 \\
5 & 3.8198 & 3.9270 & 3.9659 & 3.9835 \\
6 & 3.8556 & 3.9387 & 3.9710 & 3.9860 \\
\hline
\end{tabular}

TABLE 5.5

$H^{5}$-solution; order of convergence of the $\mathcal{A}$-semi-norm of the error in $(\boldsymbol{q}, u)$.

\begin{tabular}{c|c|c|c|c}
\hline$k$ & \multicolumn{4}{|c}{ order of convergence } \\
\hline 1 & 1.1492 & 0.9841 & 0.9905 & 0.9993 \\
2 & 1.7717 & 1.8698 & 1.9498 & 1.9828 \\
3 & 2.5591 & 2.8822 & 2.9641 & 2.9882 \\
4 & 3.7747 & 3.8689 & 3.9042 & 3.9227 \\
5 & 3.9220 & 3.9483 & 3.9710 & 3.9848 \\
6 & 3.9801 & 3.9664 & 3.9776 & 3.9876 \\
\hline
\end{tabular}

TABLE 5.6

$H^{5}$-solution; order of convergence of the $L^{2}$ error in the potential $u$.

\begin{tabular}{c|c|c|c|c}
\hline$k$ & \multicolumn{4}{|c}{ order of convergence } \\
\hline 1 & 2.1062 & 2.0203 & 2.0035 & 2.0017 \\
2 & 2.8861 & 2.9474 & 2.9686 & 2.9810 \\
3 & 3.8682 & 3.9669 & 3.9877 & 3.9945 \\
4 & 4.8685 & 4.8948 & 4.9095 & 4.9167 \\
5 & 5.0664 & 5.0310 & 5.0246 & 5.0088 \\
6 & 5.1626 & 5.0583 & 5.1051 & 5.0252 \\
\hline
\end{tabular}


5.3. Smooth solution on an L-shaped domain. We solve the model problem (1.1) in an L-shaped domain with Dirichlet boundary conditions. The exact solution is the function $u_{\alpha}$, described above, with $\alpha=4.5$. For this test we use a sequence of unstructured meshes, created from a global refinement of an unstructured coarse mesh. The number of elements of the meshes are 22, 88, 352, 1408 and 5632 .

In Tables 5.7, 5.8 and 5.9 below, we can see that we obtain the same order of convergence as in the convex case even though the standard elliptic regularity result guarantees an order of convergence for the $\mathrm{L}^{2}$-error of the potential smaller by $1-2 \gamma=\frac{1}{3}$, as indicated in Table 4.1.

A similar phenomenon takes place with the very smooth solution from the first test.

TABLE 5.7

$H^{5}$-solution on L-shaped domain; order of convergence of the $L^{2}$ error in the gradient $\boldsymbol{q}$.

\begin{tabular}{c|c|c|c|c}
\hline$k$ & \multicolumn{4}{|c}{ order of convergence } \\
\hline 1 & 0.8494 & 0.8581 & 0.9148 & 0.9530 \\
2 & 1.7966 & 1.8441 & 1.9136 & 1.9550 \\
3 & 2.6595 & 2.8369 & 2.9260 & 2.9644 \\
4 & 2.6559 & 3.7667 & 3.8908 & 3.9571 \\
5 & 2.7630 & 3.7978 & 3.8723 & 3.8912 \\
6 & 3.0742 & 3.9120 & 4.0307 & 4.1347 \\
\hline
\end{tabular}

TABle 5.8

$H^{5}$-solution on L-shaped domain; order of convergence of the $\mathcal{A}$-semi-norm of the error in $(\boldsymbol{q}, u)$.

\begin{tabular}{c|c|c|c|c}
\hline$k$ & \multicolumn{4}{|c}{ order of convergence } \\
\hline 1 & 1.0085 & 0.9079 & 0.9315 & 0.9593 \\
2 & 1.9217 & 1.8908 & 1.9295 & 1.9609 \\
3 & 2.7984 & 2.8763 & 2.9379 & 2.9688 \\
4 & 3.2364 & 3.7850 & 3.8960 & 3.9589 \\
5 & 3.7948 & 3.8105 & 3.8749 & 3.8918 \\
6 & 4.0916 & 3.9158 & 4.0313 & 4.1347 \\
\hline
\end{tabular}

TABle 5.9

$H^{5}$-solution on L-shaped domain; order of convergence of the $L^{2}$ error in the potential $u$.

\begin{tabular}{c|c|c|c|c}
\hline$k$ & \multicolumn{4}{|c}{ order of convergence } \\
\hline 1 & 2.0435 & 1.9542 & 1.9552 & 1.9714 \\
2 & 3.0471 & 2.9694 & 2.9740 & 2.9844 \\
3 & 4.0360 & 3.9693 & 3.9831 & 3.9916 \\
4 & 5.0226 & 4.8793 & 4.9274 & 4.9528 \\
5 & 5.9726 & 4.8779 & 4.8875 & 4.8739 \\
6 & 6.3544 & 4.9983 & 5.0609 & 5.0898 \\
\hline
\end{tabular}


5.4. Non-smooth solution on an L-shaped domain. Finally, we present numerical results for the classical L-shaped domain test with a singularity at the reentrant corner. We consider the model problem (1.1) in an L-shaped domain with zero right hand side and Dirichlet boundary conditions such that the exact solution is given by

$$
u(r, \theta)=r^{\gamma} \sin (\gamma \theta), \quad \gamma=\frac{2}{3} .
$$

For conforming finite element methods, it has been shown that the orders of convergence in the $\mathrm{H}^{1}$ and $\mathrm{L}^{2}$ norms are $\frac{2}{3}-\varepsilon$ and $\frac{4}{3}-\varepsilon$ for all $\varepsilon>0$, respectively. The numerical results for the LDG method on the sequence of unstructured meshes described in the previous experiment are reported in Tables 5.10, 5.11 and 5.12. They show that the rates of convergence predicted by Table 4.2 are achieved by the LDG method. Observe that the same rates of convergence as in the conforming case are achieved.

TABLE 5.10

Non-smooth solution on L-shaped domain; $L^{2}$ error in the gradient $\boldsymbol{q}$.

\begin{tabular}{c|c|c|c|c}
\hline$k$ & \multicolumn{4}{|c}{ order of convergence } \\
\hline 1 & 0.7818 & 0.6298 & 0.6420 & 0.6513 \\
2 & 0.7794 & 0.6662 & 0.6665 & 0.6666 \\
3 & 0.7362 & 0.6665 & 0.6666 & 0.6666 \\
4 & 0.7139 & 0.6666 & 0.6666 & 0.6667 \\
5 & 0.7016 & 0.6666 & 0.6666 & 0.6667 \\
6 & 0.6941 & 0.6666 & 0.6666 & 0.6667 \\
\hline
\end{tabular}

TABLE 5.11

Non-smooth solution on L-shaped domain; $\mathcal{A}$-semi-norm of the error in $(\boldsymbol{q}, u)$.

\begin{tabular}{c|c|c|c|c}
\hline$k$ & \multicolumn{4}{|c}{ order of convergence } \\
\hline 1 & 0.8043 & 0.6529 & 0.6538 & 0.6572 \\
2 & 0.7918 & 0.6766 & 0.6718 & 0.6693 \\
3 & 0.7448 & 0.6725 & 0.6696 & 0.6682 \\
4 & 0.7200 & 0.6705 & 0.6686 & 0.6676 \\
5 & 0.7062 & 0.6694 & 0.6681 & 0.6674 \\
6 & 0.6977 & 0.6688 & 0.6677 & 0.6672 \\
\hline
\end{tabular}

TABLE 5.12

Non-smooth solution on $L$-shaped domain; $L^{2}$ error in the potential $u$.

\begin{tabular}{c|c|c|c|c}
\hline$k$ & \multicolumn{4}{|c}{ order of convergence } \\
\hline 1 & 1.6098 & 1.5694 & 1.5793 & 1.5760 \\
2 & 1.5610 & 1.5383 & 1.5014 & 1.4639 \\
3 & 1.5015 & 1.4810 & 1.4449 & 1.4137 \\
4 & 1.4715 & 1.4543 & 1.4215 & 1.3950 \\
5 & 1.4535 & 1.4383 & 1.4083 & 1.3849 \\
6 & 1.4408 & 1.4277 & 1.3998 & 1.3786 \\
\hline
\end{tabular}


6. Concluding remarks. In this paper, we present the first a priori error analysis for a general DG method that includes the LDG method and allows for triangulations with hanging nodes and elements of several shapes.

We have proven that the orders of convergence of the approximations given by the LDG method with the stabilization parameter $C_{11}$ of order $h^{-1}$ are optimal; these results have been confirmed by our numerical experiments which also indicate that the quality of the approximation does not deteriorate when $C_{11}$ is taken to be of order one. Theoretically, a loss of $1 / 2$ in the orders of convergence can take place but this phenomenon was not observed in the particular test problems we considered; as a consequence, the sharpness of our error estimates in this case remains to be studied. We have also theoretically shown that the effect of taking non-zero stabilization parameters $C_{22}$ does not significantly improve the orders of convergence of the LDG method. An exception is, of course, the piecewise constant case in which the LDG method has an order of convergence of 0 whereas the DG method with $C_{11}$ and $C_{22}$ of order one do converge with orders of convergence of at least $1 / 2$ and 1 in the error of the gradient and potential, respectively.

In this paper, nothing has been said about how to chose the parameters $\boldsymbol{C}_{12}$. In a forthcoming paper [22], it will be shown that, in the case of Cartesian grids and tensor product polynomials, the orders of convergence of the LDG method can actually increase if $\boldsymbol{C}_{12}$ is suitably chosen.

Let us end by pointing out tha the implementation of codes for $h p$-adaptive versions of the LDG method for general elliptic and transient convection-diffusion-reaction problems is the subject of ongoing work.

\section{REFERENCES}

[1] D. Arnold, F. Brezzi, B. Cockburn, and D. Marini, Discontinuous Galerkin methods for elliptic problems, First International Symposium on Discontinuous Galerkin Methods (B. Cockburn, G.E. Karniadakis, and C.-W. Shu, eds.), Lecture Notes in Computational Science and Engineering, vol. 11, Springer Verlag, February 2000, pp. 89-101.

[2] D.N. Arnold, An interior penalty finite element method with discontinuous elements, SIAM J. Numer. Anal. 19 (1982), 742-760.

[3] I. Babuška and M. Zlámal, Nonconforming elements in the finite element method with penalty, SIAM J. Numer. Anal. 10 (1973), 863-875.

[4] G.A. Baker, Finite element methods for elliptic equations using nonconforming elements, Math. Comp. 31 (1977), 45-59.

[5] G.A. Baker, W.N. Jureidini, and O.A. Karakashian, Piecewise solenoidal vector fields and the Stokes problem, SIAM J. Numer. Anal. 27 (1990), 1466-1485.

[6] F. Bassi and S. Rebay, A high-order accurate discontinuous finite element method for the numerical solution of the compressible Navier-Stokes equations, J. Comput. Phys. 131 (1997), 267-279.

[7] C.E. Baumann, An hp-adaptive discontinuous Galerkin method for computational fluid dynamics, Ph.D. thesis, The University of Texas at Austin, 1997.

[8] C.E. Baumann and J.T. Oden, A discontinuous hp finite element method for the Navier-Stokes equations, 10th. International Conference on Finite Element in Fluids, 1998.

[9] C.E. Baumann and T.J. Oden, A discontinuous hp finite element method for the Euler and the Navier-Stokes equations, Int. J. Numer. Meth. Fluids (1999), to appear.

[10] R. Becker and P. Hansbo, A finite element method for domain decomposition with non-matching grids, Tech. Report 3613, INRIA, 1999.

[11] J.H. Bramble and A.H. Schatz, Higher order local accuracy by averaging in the finite element method, Math. Comp. 31 (1977), 94-111.

[12] F. Brezzi and M. Fortin, Mixed and hybrid finite element methods, Springer Verlag, 1991.

[13] F. Brezzi, D. Marini, P. Pietra, and A. Russo, Discontinuous finite elements for diffusion problems, Numerical Methods for Partial Differential Equations (1999), submitted.

[14] P. Castillo, An optimal error estimate for the local discontinuous Galerkin method, First Inter- 
national Symposium on Discontinuous Galerkin Methods (B. Cockburn, G.E. Karniadakis, and C.-W. Shu, eds.), Lecture Notes in Computational Science and Engineering, vol. 11, Springer Verlag, February 2000, pp. 285-290.

[15] P. Castillo, B. Cockburn, D. Schötzau, and Ch. Schwab, An optimal a priori error estimate for the hp-version of the local discontinuous Galerkin method for convection-diffusion problems, IMA Research Report 1689, University of Minnesota, 2000, submitted.

[16] P. Ciarlet, The finite element method for elliptic problems, North Holland, 1978.

[17] B. Cockburn, The devising of discontinuous Galerkin methods for non-linear hyperbolic conservatin laws, Journal of Computational and Applied Mathematics 00 (0000), 000-000, to appear.

[18] B. Cockburn and C. Dawson, Some extensions of the local discontinuous Galerkin method for convection-diffusion equations in multidimensions, Tech. Report Tech. Report 99-27, Texas Institute for Computational and Applied Mathematics, 1999.

[19] B. Cockburn, S. Hou, and C.W. Shu, TVB Runge-Kutta local projection discontinuous Galerkin finite element method for conservation laws IV: The multidimensional case, Math. Comp. 54 (1990), 545-581.

[20] B. Cockburn, G.E. Karniadakis, and C.-W. Shu (eds.), First international symposium on discontinuous Galerkin methods, Lecture Notes in Computational Science and Engineering, vol. 11, Springer Verlag, February 2000.

[21] B. Cockburn, S.Y. Lin, and C.W. Shu, TVB Runge-Kutta local projection discontinuous Galerkin finite element method for conservation laws III: One dimensional systems, J. Comput. Phys. 84 (1989), 90-113.

[22] B. Cockburn, I. Perugia, and D. Schötzau, Superconvergence of the local discontinuous Galerkin method for elliptic problems on Cartesian grids, in preparation.

[23] B. Cockburn and C.W. Shu, TVB Runge-Kutta local projection discontinuous Galerkin finite element method for scalar conservation laws II: General framework, Math. Comp. 52 (1989), 411-435.

[24] _ The Runge-Kutta local projection $P^{1}$-discontinuous Galerkin method for scalar conservation laws, RAIRO Modél. Math. Anal.Numér. 25 (1991), 337-361.

[25] _ The local discontinuous Galerkin finite element method for convection-diffusion systems, SIAM J. Numer. Anal. 35 (1998), 2440-2463.

[26] — The Runge-Kutta discontinuous Galerkin finite element method for conservation laws V: Multidimensional systems, J. Comput. Phys. 141 (1998), 199-224.

[27] J. Douglas, Jr. and T. Dupont, Interior penalty procedures for elliptic and parabolic Galerkin methods, Lecture Notes in Physics, vol. 58, Springer-Verlag, Berlin, 1976.

[28] P. Grisvard, Elliptic problems in nonsmooth domains, Monographs and Studies in Mathematics, 34, Pitman, Boston, 1985.

[29] Singularities in boundary value problems, Research Notes in Applied Mathematics, Masson, Paris, 1992.

[30] C. Johnson and J. Pitkäranta, An analysis of the discontinuous Galerkin method for a scalar hyperbolic equation, Math. Comp. 46 (1986), 1-26.

[31] J.T. Oden, Ivo Babuška, and C.E. Baumann, A discontinuous hp finite element method for diffusion problems, J. Comput. Phys. 146 (1998), 491-519.

[32] T.J. Oden and C.E. Baumann, A conservative D GM for convection-diffusion and Navier-Stokes problems, First International Symposium on Discontinuous Galerkin Methods (B. Cockburn, G.E. Karniadakis, and C.-W. Shu, eds.), Lecture Notes in Computational Science and Engineering, vol. 11, Springer Verlag, February 2000, pp. 179-196.

[33] T. Peterson, A note on the convergence of the discontinuous Galerkin method for a scalar hyperbolic equation, SIAM J. Numer. Anal. 28 (1991), 133-140.

[34] B. Rivière and M.F. Wheeler, A discontinuous Galerkin method applied to nonlinear parabolic equations, First International Symposium on Discontinuous Galerkin Methods (B. Cockburn, G.E. Karniadakis, and C.-W. Shu, eds.), Lecture Notes in Computational Science and Engineering, vol. 11, Springer Verlag, February 2000, pp. 231-244.

[35] B. Rivière, M.F. Wheeler, and V. Girault, Improved energy estimates for interior penalty, constrained and discontinuous Galerkin methods for elliptic problems. Part I, Tech. Report 99-09, TICAM, 1999.

[36] T. Rusten, P.S. Vassilevski, and R. Winther, Interior penalty preconditioners for mixed finite element approximations of elliptic problems, Math. Comp. 65 (1996), 447-466.

[37] A.H. Schatz and L.B. Wahlbin, Maximum norm estimates in the finite element method in plane polygonal domains. Part I, Math. Comp. 32 (1977), 73-109.

[38] E. Süli, Ch. Schwab, and P. Houston, hp-DGFEM for partial differential equations with nonnegative characteristic form, First International Symposium on Discontinuous Galerkin 
Methods (B. Cockburn, G.E. Karniadakis, and C.-W. Shu, eds.), Lecture Notes in Computational Science and Engineering, vol. 11, Springer Verlag, February 2000, pp. 221-230.

[39] M.F. Wheeler, An elliptic collocation-finite element method with interior penalties, SIAM J. Numer. Anal. 15 (1978), 152-161.

[40] T. Wihler and C. Schwab, Exponential convergence of the hp-DGFEM for diffusion problems in two space dimensions, Preprint, in preparation.

[41] _ Robust exponential convergence of the hp discontinuous Galerkin FEM for convectiondiffusion problems in one space dimension, East West J. Num. Math., to appear. 